\title{
The origin of the metal enrichment of carbon nanostructures produced by laser ablation of a carbon-nickel target
}

\author{
J. Fryar, K. D. G. I. Jayawardena, S. R. P. Silva, and S. J. Henley ${ }^{*}$ \\ Nano-Electronics Centre, Advanced Technology Institute, University of Surrey, GU2 7XH, United Kingdom
}

Compositional analysis of metal-containing carbon thin films and nanostructures produced by pulsed laser ablation of a carbon-nickel target revealed significantly higher fractions of nickel in the materials than in the target used to produce them. Ablation of mixed targets is used routinely in the synthesis of carbon nanotubes and to enhance the conductivity of amorphous carbon films by metal incorporation. In this extensive study we investigate the physical mechanisms underlying this metal-enrichment and relate changes in the dynamics of the ablation plumes with increasing background gas pressure to the composition of deposited materials. The failure to preserve the target atom ratios cannot, in this case, be attributed to conventional mechanisms for non-stoichiometric transfer. Instead, nickel-enrichment of the target surface by back-deposition, combined with significantly different propagation dynamics for $\mathrm{C}$ atoms, $\mathrm{Ni}$ atoms and alloy clusters through the background gas, appears to be the cause of the high nickel fractions.

\section{Introduction}

The pulsed laser ablation of mixed carbon-metal targets is used extensively in the catalytic growth of carbon nanostructures such as carbon nanotubes and to produce conductive amorphous carbon/metal alloy film. The authors have reported previously significant enhancements in the electrical properties of nanostructured carbon films deposited by laser ablation of mixed C:Ni targets in background pressures of argon gas [1]. Allotropes of carbon 
formed through cluster assembly [2,3] typically display high porosities and large surface areas [4,5], with $s p^{3}$ bond formation at the surface of clusters limiting the conductivity [6], and the observed improvements in electrical characteristics were therefore attributed to a preferential incorporation of $\mathrm{Ni}$ in the films and an incongruent transfer of material from target to substrate.

The behaviour of mixed targets has received much attention, particularly in relation to PLD growth of complex oxide high- $T_{C}$ superconductors and semi-magnetic semiconductor films [7-10]. For these composite systems small deviations from the target stoichiometry in deposited layers has been attributed to differences in the thermal properties of the target constituents [11], differing angular distributions of the ablation products [12], and sputtering effects at the substrate itself [13]. Since a 30 or $45^{\circ}$ angle of incidence is generally used for ablation, the on-target laser spot tends to be elliptical particularly for excimer lasers with spatially broad output beams. This can lead to the so-called 'flip-over' effect whereby deposited material has an elliptical distribution on the substrate, but with major and minor axes rotated at $90^{\circ}$ relative to the spot $[14,15]$. For multi-component targets, this flip-over effect may also result in an incongruent transfer of material [16].

Here, plumes accompanying ablation of the mixed carbon-nickel target [1] are examined using optical emission spectroscopy and filtered imaging in an attempt to understand the impact on both plume dynamics and sample composition of varying the background gas pressure. While the focus of the current research is primarily to understand the reasons for the high Ni:C ratio, these mixed plumes offer a somewhat unique system for more general studies of plume expansion in inert gases, particularly since $\mathrm{C}$ is lighter and Ni heavier than 
the background gas. In addition, the discussion presented is of direct relevance the ablation of mixed C-Ni-Co targets used for carbon nanotube growth.

\section{Experimental Details}

A mixed carbon-nickel sintered target containing 20 at\% Ni (PI-KEM: Lot\#12295S-22101204) was ablated at a $45^{\circ}$ angle of incidence using a $\mathrm{KrF}$ excimer laser (Lambda Physik LPX210i) at $248 \mathrm{~nm}$ with a FWHM pulse duration of $\sim 25 \mathrm{~ns}$. In all cases the fluence was maintained at $\sim 6 \mathrm{~J} / \mathrm{cm}^{2}$ over an elliptical laser spot of area $\sim 1 \mathrm{~mm}^{2}$ (major axis twice as long as minor axis) and the target rotated to prevent target erosion. For film depositions a rotation rate producing a linear speed $2 \mathrm{~mm} / \mathrm{s}$ at the spot and a pulse repletion rate of $10 \mathrm{~Hz}$ were chosen such that each laser spot overlapped $60 \%$ with the previous one. For spectroscopy the pulse repletion rate was decreased to $1 \mathrm{~Hz}$, removing the overlap. Separate pyrolytic graphite and nickel targets were also ablated under identical conditions to allow a comparison of plume optical emission characteristics.

Images of the plume were captured using a $1024 \times 1024$ pixel Andor iStar camera (DH73418U-03), which was triggered by the HV charging output of the laser. Jitter has been measured to be approximately $\pm 1 \mathrm{~ns}$ at a repetition rate of $1 \mathrm{~Hz}$. To avoid saturation of the CCD by intense nickel emission, plumes have been imaged through a neutral density filter $(\mathrm{OD}=2$ @ $550 \mathrm{~nm})$. This was subsequently replaced with band pass filters to examine relevant plume components and their optical characteristics are discussed where appropriate. Emission was focused on the camera using a biconvex lens and resolution is $\sim 55 \mu \mathrm{m}$ per pixel. The camera viewing direction, in all cases, is perpendicular to the target surface normal.

Optical emission spectra (OES) at different distances from the target were obtained by translating a fibre along the plume propagation direction. Captured light was dispersed by an Andor Shamrock 303i spectrograph containing a 300 lines/mm grating, blazed at $300 \mathrm{~nm}$. 
The Andor camera was used as detector in this configuration and the resolution of the obtained spectra is $\sim 0.1 \mathrm{~nm}$. After focusing optics, the fibre observes plume emission over a $\sim 3 \mathrm{~mm}$ diameter and values given in the text for its position represent the centre of this fieldof-view. All quoted time intervals are measured from the moment laser light is detected ontarget using the minimum camera gate width of $\sim 2 \mathrm{~ns}$.

Two-inch diameter silicon wafers were used as a substrate for material growth and were positioned $6 \mathrm{~cm}$ from the target. This distance was chosen to match that of a previous study of graphite ablation [17]. Films were deposited in different background pressures of argon gas, referred to as $p(\mathrm{Ar})$ within the text, to investigate the effects of plume confinement. The base pressure of the chamber was $\sim 5 \mu$ Torr $\left(6.7 \times 10^{-6}\right.$ mbar $)$ and gas pressure was controlled using a mass-flow controller and needle valve. The relative Ni concentration in the films has been determined using energy dispersive x-ray spectroscopy (EDX) (Oxford INCA Penta FETx3) at a number of locations for each sample grown at each pressure. EDX data from 20 $\mu \mathrm{m}^{2}$ areas confirms that the $\mathrm{C}: \mathrm{Ni}$ fraction is uniform over the cleaned target surface before ablation and the EDX results from the target were subsequently used as a reference to calibrate the $\mathrm{C}$ to $\mathrm{Ni}$ ratio. The accuracy of the EDX data has been confirmed by $\mathrm{X}$-ray photoelectron spectroscopy on a number of the generated C-Ni films. Sample and target morphology were examined using Philips XL30 and FEI Quanta 200F scanning electron microscopes (SEM).

\section{Results and Discussion}

\subsection{EDX and SEM analysis of substrates}

To briefly summarize the results previously reported in [1], EDX data for samples deposited at $p(\mathrm{Ar})=5,40,100$ and $340 \mathrm{mT}\left(\sim 6.7,53,133\right.$ and $453 \times 10^{-3}$ mbar $)$ reveals that the 
concentration of $\mathrm{Ni}$ in films is substantially higher than the 20 at $\%$ within the target material. The measured Ni fraction is $\sim 52$ at $\%$ in the film region directly opposite the on-target laser spot $(x, y=0)$ with a $5 \mathrm{mT}$ background pressure, and is found to decrease to $\sim 43 \%$ at $40 \mathrm{mT}$ and $\sim 39 \%$ at $100 \mathrm{mT}$. When $p(\mathrm{Ar})$ is increased to $340 \mathrm{mT}$, Ni concentration recovers to 46 at\%. Although the data from [1] show a slight angular dependence of the Ni:C ratio, the relative reduction in the $\mathrm{Ni}$ fraction is at most 5 at $\%$ at sample locations $x, y= \pm 1.5 \mathrm{~cm}$ from the centre position. One can therefore conclude that the $\mathrm{C}$ :Ni ratio in the thin films is roughly uniform over a surface area of $\sim 4.5 \mathrm{~cm}^{2}$.

SEM micrographs of the films deposited at each background pressure are shown in Figures 1(a) to 1(d). Further details, including Raman analysis, can be found in [1]. The observed change in morphology as a function of $p(\mathrm{Ar})$ is similar to that reported for films grown by UV laser ablation of pyrolytic graphite targets [17,18] where smooth $s p^{3}$ rich films are deposited at low pressures, as in Figures 1(a) and (b), due to local densification by the impact of high energy $\mathrm{C}$ atoms/ions [19]. At higher pressures, Figures 1(c) and (d), confinement leads to a collision-induced formation of clusters within the plume [2]. A comparison between the C:Ni films and carbon films studied in [17] suggests that clustered material occurs at a higher pressure in the mixed target case $(\sim 100 \mathrm{mT})$ than for a graphite target $(\sim 40$ mT) under similar ablation conditions, implying that the carbon yield per laser shot may be substantially reduced when using the mixed target. 

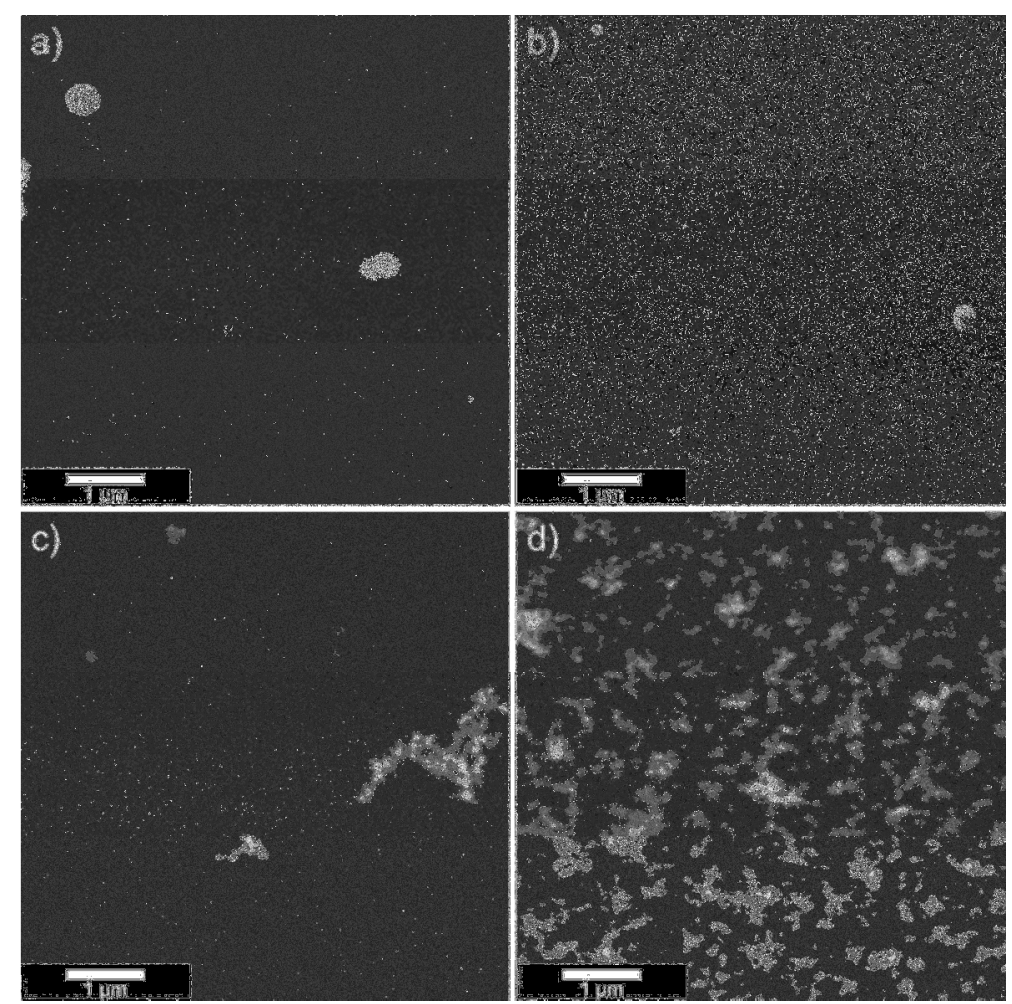

Figure 1: SEM micrographs of C-Ni thin films grown at (a) 5, (b) 40, (c) 100, and (d) $340 \mathrm{mT}$ Ar pressure. Films grown at low pressures, (a) and (b), are similar to the smooth $s p^{3}$-rich films deposited at low pressures using graphite targets. At higher pressures, (c) and (d), confinement of the plume leads to the formation of clustered material.

The $\sim 52: 48 \% \mathrm{Ni}: \mathrm{C}$ ratio in films deposited at $5 \mathrm{mT}$ was found to be equivalent to the ratio in samples grown at the base pressure of the deposition system (i.e. in the absence of the background gas). One possible explanation for the high nickel fraction in the films is that the angular distribution of carbon and nickel species within the plume is different, with carbon having the broader distribution and nickel being highly forward-focused. Such angular distributions are typically described by a $\cos ^{n} \theta$ function, with $\theta$ representing the angle toward the normal of the target surface and the exponent $n$ determined by a fit to sample thickness and lying in the range $\sim 4$ to 30 [20]. Since the target-to-substrate distance in this case is $6 \mathrm{~cm}$ and the EDX results show the film composition to be relatively uniform over a distance of $\pm 1.5 \mathrm{~cm}$, the subtended angle from the target normal to the edge of the EDX- 
examined region is $\sim 14^{\circ}$. Assuming that nickel and carbon species will have different angular distributions and hence different values of exponent $n$, and that the ablation yield matches the 80:20 at\% C:Ni ratio of the target, a simple numerical analysis reveals that there is no combination of $n$ values in the range 1 to 40 that can achieve the observed 52:48\% ratio in samples. This suggests that the high nickel fraction found in the films grown under vacuum and low pressure conditions is primarily due to a higher yield of Ni than expected, rather than the angular distribution of species.

In previous work, the composition of films grown at $p(\mathrm{Ar})=5 \mathrm{mT}$ using an ArF laser at 193 $\mathrm{nm}$ (with a much higher target rotation rate starting with a clean target, but subsequently reusing the ablated area for later samples) rather than the $\mathrm{KrF}$ laser and the slow rotation rates discussed here, was found to be close to the 80:20 at\% C:Ni ratio of the target [1] However, the Nickel content of subsequently deposited films at higher pressures was unpredictable. ArF ablation of graphite targets tends to produce a higher proportion of ionized carbon and higher forward velocities of plume components than $\mathrm{KrF}$ ablation, which is also reflected in the increased $s p^{3}: s p^{2}$ bonding ratio in films grown by ArF laser deposition [21]. The nearcongruent transfer of material in the ArF case demonstrates that sputtering of the film cannot be the dominant mechanism for metal enrichment, as the energy of the growth species would be expected to be higher here and cause more not less sputtering. The fact that at higher argon pressures the Ni remains in excess, also suggests that sputtering of the target is not the most significant effect as the emitted species will be entirely thermalized well before they reach the target at 340 mTorr. We therefore conclude that the high nickel content in films deposited at low pressures using the $\mathrm{KrF}$ laser is also unlikely to be due to the removal of material (particularly carbon) by sputtering. The discrepancy in the Ni content of the two sets of films, 
is due to different ablation parameters, particularly as we reuse the same target surface for growing the higher pressure films in the ArF case, which will be discussed in detail later.

\subsection{Examination of the target pre- and post-ablation}

CCD imaging of the ablation plumes, to be discussed later, also captures optical emission from the heated target surface after the initial laser interaction. Figure 2 shows a comparison of the surface emission intensity (integrated over visible wavelengths) as a function of time for both pyrolytic graphite and mixed carbon-nickel targets after $\mathrm{KrF}$ ablation under identical conditions with $p(\mathrm{Ar})=40 \mathrm{mT}$. The graphite target emission is found to decrease rapidly, which is interpreted as a shift to IR wavelengths beyond those amplified by the camera's intensifier, whereas the carbon-nickel target emission persists in the visible region for several microseconds. This data indicates that there is a substantial difference in the relative ability of each target to dissipate heat away from the ablation zone, due to differences in their thermal conductivities.

Figures 3(a) and 3(b) are, respectively, 25 and $12.5 \mu \mathrm{m}^{2} \mathrm{SEM}$ images of the mixed target surface before ablation and show it to be composed of overlapping graphite flakes, with dimensions on the order of a few microns, interspersed with 'cubic' $\mathrm{Ni}$ regions. A comparison of the mixed target mass before and after multiple ablation events in vacuum with high target rotation rates indicates the yield is $\sim 0.5 \mu \mathrm{g}$ per laser shot. This mass loss corresponds to $\sim 1 \times 10^{16}$ atoms of carbon and $\sim 3 \times 10^{15}$ atoms of nickel if it is assumed for the moment that the 80:20 at\% C:Ni ratio of the target is maintained in the resulting plume. For comparison, the yield from a pyrolytic graphite target under identical experimental parameters is $\sim 1 \mu \mathrm{g}$ or $\sim 5 \times 10^{16}$ atoms of carbon. As a rough estimate, one would expect the carbon yield from the mixed target to be at most $20 \%$ of that from a pyrolytic graphite target 
per laser shot. The disordered nature of the starting material and a significantly reduced carbon yield may therefore explain the higher pressures required in the mixed target case to observe clustering in films with respect to [17], as discussed previously. Furthermore, since the thermal diffusivity of graphite decreases by several orders of magnitude with increasing crack density [22], and conductivity decreases as a function of porosity [23], the relatively slow cooling of the mixed target is also consistent with its structure as it's thermal conductivity is expected to must lower than the dense pyrolytic graphite target.

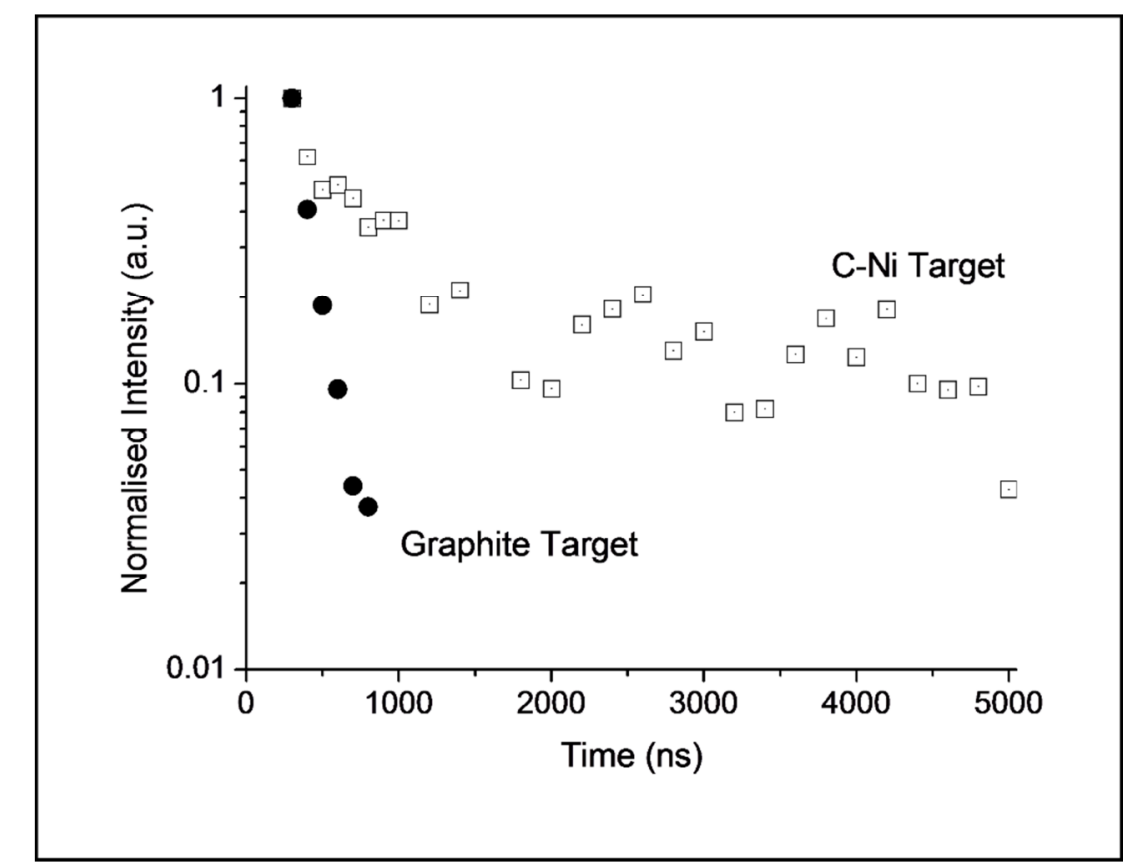

Figure 2: Decay rate of the integrated intensity of light in visible wavelengths emitted from the target ablation zone extracted from spectra obtained from the Shamrock spectrometer. The graphite target appears to cool significantly faster than the mixed target. The sinusoidal pattern in the $\mathrm{C}-\mathrm{Ni}$ data from $1000 \mathrm{~ns}$ onwards is due to slight variations in the target position relative to the camera as it rotates. 

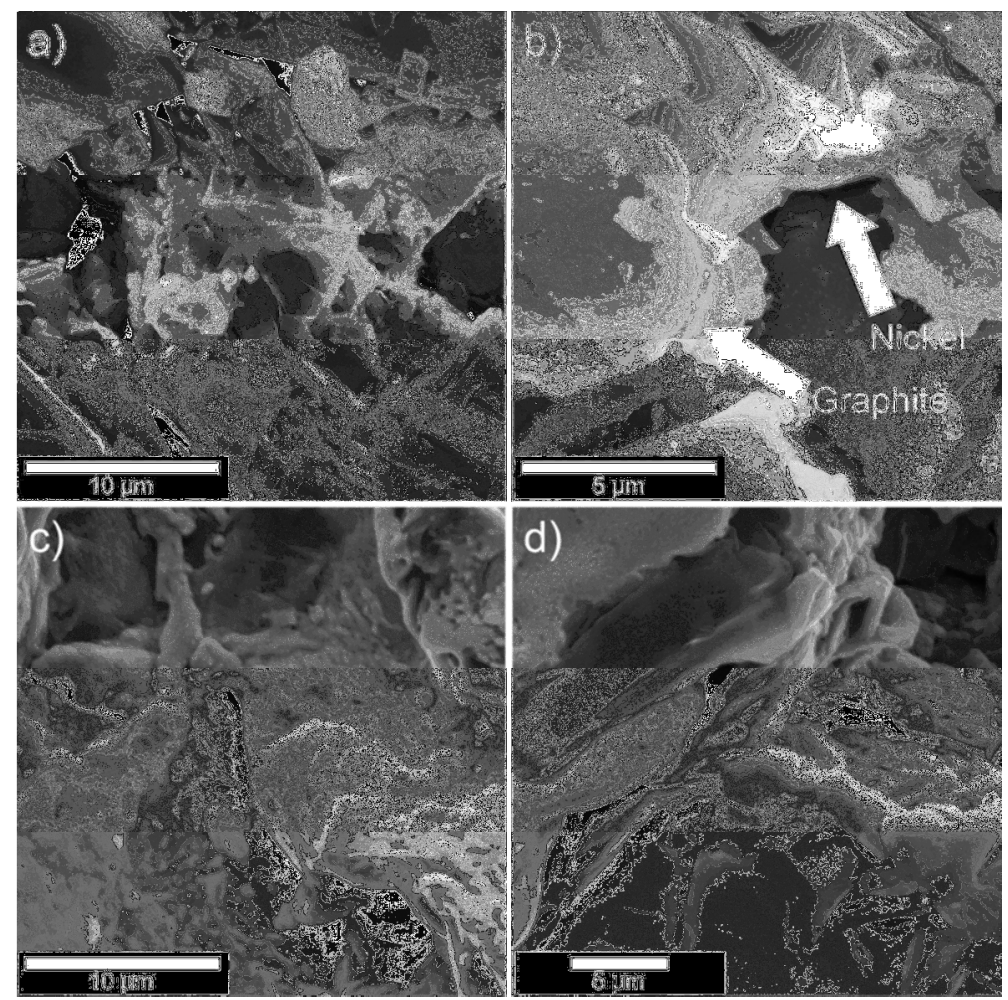

Figure 3: SEM images of the target before and after $\mathrm{KrF}$ laser ablation. The pre-ablated material is highly disordered and porous (a), and composed of individual graphite flakes and cubic nickel regions (b). The post-ablation surface (c) shows a significant change in morphology with evidence of liquid-phase flow, and regions outside the ablation zone, although not directly illuminated by the laser spot, also show evidence of morphological change (d).

Figure 3(c) is a $25 \mu^{2}$ SEM micrograph from within the ablated target region after a deposition run in vacuum. A significant morphological change of material occurs with evidence of appreciable melting and subsequent flow of liquid-phase material. This observation is explained by an accompanying EDX analysis that shows Ni concentrations as high as $\sim 76$ at $\%$. Figure $3(\mathrm{~d})$ is an SEM image of a region several millimetres outside the ablation zone, and thus far removed from direct illumination by the laser spot. The target surface at such locations also display evidence of thermal modification and EDX data 
accordingly shows that the $\mathrm{Ni}$ fraction is substantially higher than the $20 \%$ value before ablation.

One possible mechanism for the nickel-enrichment of the target surface may be related to its post-ablation thermal behaviour rather than a preferential removal of carbon during the laser pulse. It is difficult to reconcile a preferential removal of one species relative to another with the $6 \mathrm{~J} / \mathrm{cm}^{2}$ fluence utilised in this study since this is above the threshold required to produce so-called 'phase explosion' in both graphite [24] and nickel [25] targets. There is also no evidence from spectroscopic or imaging data to suggest a carbon-rich plume is generated after a single laser shot. Given that nickel has a high vapour pressure (237 $\mathrm{Pa}$ at $1726 \mathrm{~K}$ ), and that the heat of vaporization of nickel $\left(377.5 \mathrm{~kJ} \mathrm{~mol}^{-1}\right)$ is substantially lower than the heat of sublimation of graphite $\left(716.7 \mathrm{~kJ} \mathrm{~mol}^{-1}\right)$, it is suggested that these thermal properties may favour the migration of nickel to the target surface during its prolonged post-ablation cooling.

Hence, an initial candidate for the explanation behind the high nickel fraction found in thin films deposited at $p(\mathrm{Ar}) \leq 5 \mathrm{mT}$ is that the $60 \%$ spot overlap results in ablation of a nickelenriched surface regions created in the ablation crater of each shot. However, the EDX analysis of the target showed a significant $\mathrm{Ni}$ enrichment well away from the ablation zone, so this may not be the only mechanism. Considering the difference in atomic collision crosssections of nickel and carbon, it is suggested that nickel may be preferentially back-scattered towards the target surface. A similar mechanism has been proposed in the case of $\mathrm{ZnO}$ targets, where the $\mathrm{Zn:O}$ ratio in films is found to be greater than unity. [26] Thus, we suggest that, although a thermally-related nickel-enrichment of the target may occur at the ablation zone, that back-deposition of material from the plume may be more significant. This backdeposited material could be subsequently re-ablated, leading to a plume that becomes 
increasingly nickel-rich with increasing number of shots. We will test this hypothesis later. In the ArF case, a much higher target rotation rate was used, compared to that for the $\mathrm{KrF}$ ablation, with no spot overlap, and the first sample was deposited with a clean target, but subsequent samples re-used the same irradiated area. We believe this can finally explain the difference observed between the $\mathrm{ArF}$ and $\mathrm{KrF}$ results shown in [1].

One final mechanism for enrichment would be a change in sticking coefficients for the different species, once they reach the substrate. If Ni sticks more easily than $\mathrm{C}$ on impact, the films would appear enriched, even if the ablation was congruent. If, as the pressure changes the nature and energy of the impacting species also changes (as is observed) then the sticking coefficient would also change. Due to the complex nanostructured morphology of the sample at higher pressures, it was not possible to directly measure the thickness and hence mass of the deposited material, so directly assessing the sticking coefficients was not possible. However, it would be expected that the sticking coefficients would be independent of the laser spot overlap on the target.

To test if proposed mechanism for back-scattering induced enrichment is the most significant the composition of samples grown in vacuum conditions using the $\mathrm{KrF}$ laser at high rotation rates was measured. At an target angular velocity three times that used for the initial sample the nickel fraction is found to decrease from $52 \%$ to 46 at $\%$; at six times the rate, the relative nickel fraction dropped to $40 \%$. In both these cases there will be no overlap of laser spots on the target surface. Therefore, it is found that the composition of films gets closer to the ratio in the target for fast rotation rates. Furthermore, this dependence on target rotation rather than spot size rules out the 'flip-over' effect $[14,15,16]$ as the dominant mechanism affecting the film composition. The observation that a significant drop in the Ni content occurs by simply 
increasing the target rotation rate suggests that changes in the sticking coefficient is less important than the effect of back-deposition. A more detailed analysis of the back-scattering phenomenon through the use of collision simulations is currently underway. It should be noted that if an appreciable quantity of $\mathrm{Ni}$ is back-scattered onto the target then the above measurements of mass loss per laser shot would result in an over-estimation of the yield of carbon. This very significant effect has important implications in the catalytic growth of carbon nanostructures by pulsed laser ablation as the yield of metal in the plume is likely to be affected strongly by the experimental parameters. The spot spacing and target utilization need to be standardised and it may take many complete passes of the laser over the target for a stable ablation stoichiometry to be achieved.

\subsection{Optical emission spectroscopy}

Optical emission spectra (OES) obtained on ablation of a pyrolytic graphite, the mixed C-Ni, and a nickel target at the base pressure of the chamber are shown in Figure 4. The laser fluence in each case is $6 \mathrm{~J} / \mathrm{cm}^{2}$, the gate width $500 \mathrm{~ns}$, and the fibre positioned to observe emission $2 \mathrm{~mm}$ from the target surface. Spectra have been accumulated over 100 laser shots and acquisition began at $\mathrm{t}=0 \mathrm{~ns}$ for Fig. 4(a) and $500 \mathrm{~ns}$ for Fig. 4(b). The laser repetition rate used to acquire spectra was $1 \mathrm{~Hz}$, which is significantly lower than the $10 \mathrm{~Hz}$ rate used for the deposition of samples. The influence of re-ablated, back-scattered material on the data is therefore believed to be minimal.

From this comparison of targets it is clear that the carbon-nickel plume emission is virtually identical to that of the nickel reference, with corresponding features attributable to transitions of electronically-excited neutral $\mathrm{Ni}^{1}{ }^{1}$ Other than a significant decrease in the signal-to-noise

\footnotetext{
${ }^{1}$ NIST Atomic Spectra Database. http://www.nist.gov/pml/data/asd.cfm
} 
ratio, mixed plume spectra accumulated over 100 laser shots during the first 500 ns of plume evolution appear virtually identical to those acquired with single and small numbers of laser shots. The pair of lines labelled '?' in Fig. 4(a) at 393.4 and $396.9 \mathrm{~nm}$ are consistently observed in the mixed plume and do not correspond to either carbon or nickel transitions. On the basis of wavelength, intensity, and known impurities in the target, we attribute both to Ca. In terms of carbon emission, only broad 426.7 and weak $537.9 \mathrm{~nm}$ lines are common to both the mixed C-Ni and graphite plume in Fig. 4(a). The $426.7 \mathrm{~nm}$ feature is correlated with the $4 f^{1} ;{ }^{2} F^{\circ} \rightarrow 3 d^{1} ;{ }^{2} D$ transition of singly-ionised carbon and its intensity in the mixed plume is roughly half that found in the graphite plume. The $537.9 \mathrm{~nm}$ line is the $4 p ;{ }^{1} \mathrm{P} \rightarrow 3 s ;{ }^{1} \mathrm{P}^{\circ}$ transition of neutral carbon, with intensity in the $\mathrm{C}$-Ni case of just one-third that in the graphite plume emission. Other neutral (labelled ' $C$ ' in Fig. 4) and ionised (labelled ' $C^{+}$') carbon lines are usually detected on ablation of pyrolytic graphite $[17,27,28]$ at equivalent laser fluence but evidence for these is not apparent in the mixed plume spectra. However, it is unlikely that one could observe these specific carbon emission lines due to the overwhelming intensity of Ni-related features in equivalent wavelength regions.

$\mathrm{KrF}$ laser ablation of graphite, as also shown in Fig. 4, tends to produce the characteristic Swan-band $\left(d^{3} \Pi_{g} \rightarrow a^{3} \Pi_{u}\right)$ of $C_{2}$ molecules [17,28,29] and an intense, broad, 'blackbody-type' emission at short wavelength that has been attributed to $C_{3}$ [30], small particulates $[31,32]$, and electron bremsstrahlung. No evidence of either feature is found in the spectra of mixed carbon-nickel plumes under vacuum conditions. Based on the signal-to-noise ratio and intensity of the weakest lines, the system would fail to detect the $C_{2}$ Swan-band in the mixed plume if its emission intensity was approximately one-fifth the intensity observed for the graphite plume. 
This $20 \%$ value for $\mathrm{C}_{2}$ emission, as well as the $\sim 33 \%$ relative intensity of neutral carbon emission lines, is roughly consistent with the estimated relative carbon yield from the mixed target based on the measured mass loss per laser shot. The high intensity of the $426.7 \mathrm{~nm}$ ionised carbon emission in the mixed plume therefore appears somewhat anomalous. Song et al. have shown that, at a fluence of $\sim 6 \mathrm{~J} / \mathrm{cm}^{2}$, the transmissivity of $\mathrm{Ni}$ ablation plumes to the $248 \mathrm{~nm} \mathrm{KrF}$ line reduces to approximately $50 \%$ within $20 \mathrm{~ns}$ of the onset of the laser pulse [33]. The $3 s^{1} ; P^{\mathrm{o}} \rightarrow 2 p^{2} ;{ }^{1} S$ transition of carbon $(247.9 \mathrm{~nm})$ is close to the $\mathrm{KrF}$ wavelength and the mixed $\mathrm{C}$-Ni plume is therefore expected to be highly absorbing of incident laser radiation. The presence of short-wavelength nickel emission lines in the spectra may increase the proportion of ionised carbon as a result of subsequent multi-photon excitation processes, giving rise to enhanced emission at $426.7 \mathrm{~nm}$ with respect to overall yield. Furthermore, screening effects may be reduced since the density of carbon is relatively low compared to a graphite target.

D. L. Pappas et al. have examined $\mathrm{C}_{2}$ yields on ablation of a graphite target as a function of $\mathrm{KrF}$ laser fluence [34] and observed that yield peaks at $\sim 5 \mathrm{~J} / \mathrm{cm}^{2}$ and decreases to just $20 \%$ of maximum at $\sim 8 \mathrm{~J} / \mathrm{cm}^{2}$. This decrease was attributed to dissociation of fast moving $\mathrm{C}_{2}$ molecules by electron impact at the forward edge of the plume. The failure to detect $\mathrm{C}_{2}$ emission in the mixed target OES in vacuum, as well as the broad emission at short wavelengths (assuming it to be related to $\mathrm{C}_{3}$ ) may also then be consistent with a proportional increase in carbon ion/electron yields. 


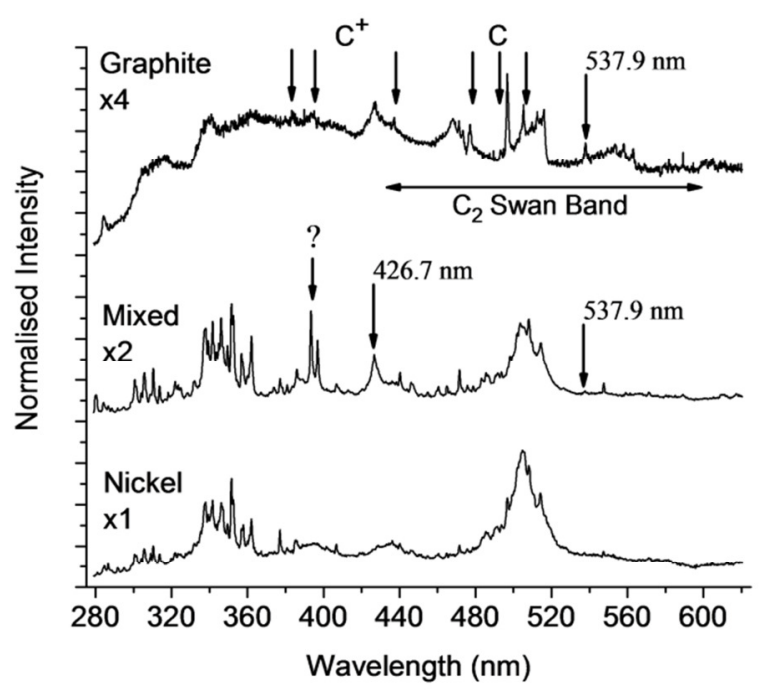

(a)

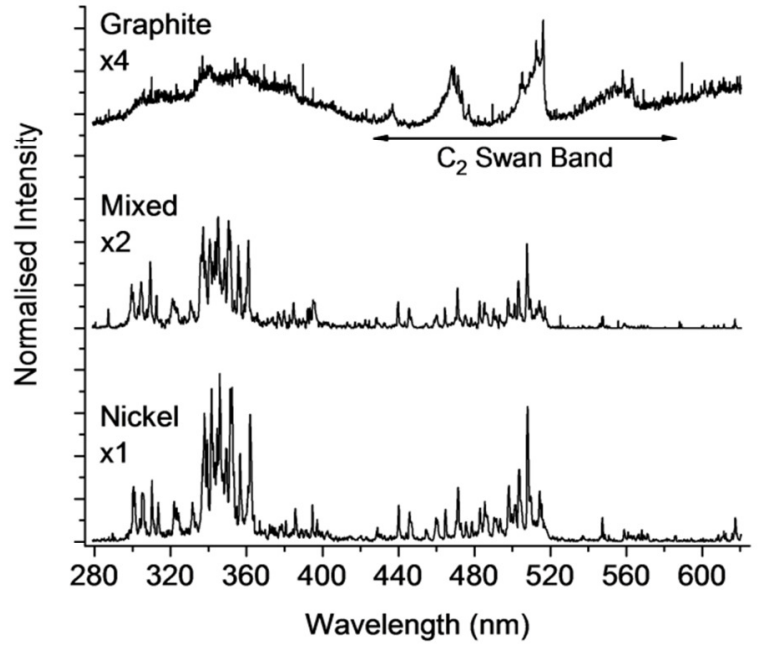

(b)

Figure 4: Optical emission spectra of graphite, mixed $\mathrm{C}-\mathrm{Ni}$, and $\mathrm{Ni}$ plumes in vacuum at time 0-500 ns (a) and 500-1000 ns (b). The distance of the fibre from the target is $2 \mathrm{~mm}$ in each case. All spectra have been normalised to account for grating, fibre, CCD and neutral density filter efficiencies, and changes in camera gain settings. Graphite and C-Ni spectra have been offset for clarity. 
To aid in the identification of features observed through plume imaging, spatially-resolved spectra of the mixed plume were acquired both in vacuum, Figures 5(a) and (b), and at $p(\mathrm{Ar})$ $=340$ mT, Figs. 5(c) and (d). As with the comparison between targets above, Figs. 5(a) and (c) were acquired between 0 and $500 \mathrm{~ns}$, and 5(b) and (d) between 500 and $1000 \mathrm{~ns}$ after the laser pulse. The plume has been examined at distances of 2, 7 and $15 \mathrm{~mm}$ along the plume propagation direction perpendicular to the target surface.

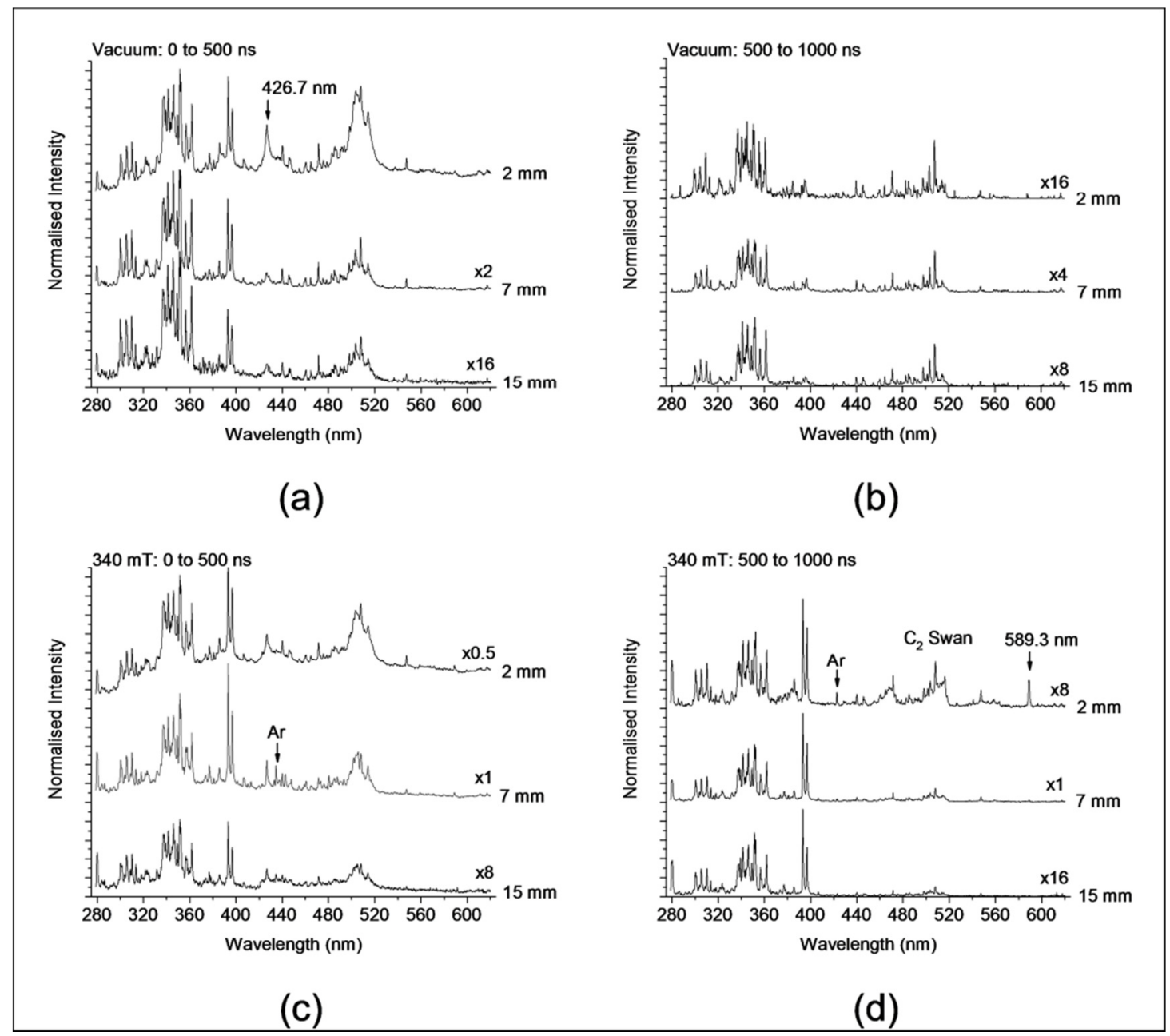

Figure 5: Optical emission spectra of mixed C-Ni plumes in vacuum $(a, b)$ and at an argon pressure of $340 \mathrm{mT}(\mathrm{c}, \mathrm{d})$. In each case the plumes have been acquired between 0-500 ns (a, c) and 500-1000 $\mathrm{ns}(\mathrm{b}, \mathrm{d})$ and examined at 2, 7 and $15 \mathrm{~mm}$ from the target. 
From Fig. 5(a), the $426.7 \mathrm{~nm} \mathrm{C}^{+}$line in vacuum can be traced out to $15 \mathrm{~mm}$ within the first $500 \mathrm{~ns}$ indicating a velocity of $\sim 30 \mathrm{~km} / \mathrm{s}$. This feature does not appear at later times, Fig. 5(b), demonstrating that ionised carbon emission is relatively short-lived and/or the majority of emitting species clear a distance of $15 \mathrm{~mm}$ within the gating period. In Fig. 5(b) the increase in $\mathrm{Ni}$ emission intensity at a distance of $7 \mathrm{~mm}$ (shown at $\times 4$ relative magnification), compared to spectra obtained at 2 and $15 \mathrm{~mm}$ (shown at $\times 16$ and $\times 8$ respectively) over the same gate period (500 to $1000 \mathrm{~ns}$ ), indicates that the bulk of observable Ni has a forward velocity somewhere between 7 and $14 \mathrm{~km} / \mathrm{s}$ in vacuum. One can hence conclude that the plume in vacuum is composed of at least two distinct emission regions: a fast moving component initially containing ionised carbon $(\sim 30 \mathrm{~km} / \mathrm{s})$, and a nickel component with a velocity of $\sim 7$ to $14 \mathrm{~km} / \mathrm{s}$.

At a background argon pressure of $340 \mathrm{mT}$, the intensity of emission in Fig. 5(c) near the target $(2 \mathrm{~mm})$ is enhanced by approximately a factor of two compared to the emission in vacuum. This enhancement in intensity is attributed to excitation due to collisions with background gas atoms and/or electron impact. Since electron-atom collision cross-sections are at least two orders of magnitude larger than those of atom-atom collisions [35], electron impact processes are expected to play a dominant role. Spectra taken over the first $500 \mathrm{~ns}$ at $340 \mathrm{mT}$ are broadly comparable to those taken in vacuum although the relatively strong singly-ionised Ar emission peak at $434.8 \mathrm{~nm}$ is now observed due to interactions between the plume and background gas.

During the period 500 to 1000 ns after the laser pulse, Fig. 5(d), a weak $\mathrm{C}_{2}$ Swan-band is evident at $2 \mathrm{~mm}$ from the target, giving a velocity of $\sim 2$ to $4 \mathrm{~km} / \mathrm{s}$ for $\mathrm{C}_{2}$ molecules. The presence of the $\mathrm{C}_{2}$ band is accompanied by a weak ionised argon line at $422.8 \mathrm{~nm}$ and neutral 
nickel emission at $589.3 \mathrm{~nm}$. The observed enhancement of $\mathrm{C}_{2}$ emission in the presence of a background gas has been attributed to a recombination of carbon atoms and ions, or dissociation of clusters [36]. In the case of the mixed plume, the intensity of the neutral nickel line at $589.3 \mathrm{~nm}$ could indicate collisions between such clusters and the tail-end of the nickel distribution. Alternatively, if the emergence of $\mathrm{C}_{2}$ is due to recombination of $\mathrm{C}$ atoms/ions then Ar and/or Ni may play a 'third-body' role in stabilising the formation.

While the spectra presented here reveal spatial separations between ionized carbon, neutral nickel, and $\mathrm{C}_{2}$ components of the mixed plume, they largely fail to isolate emission from electronically-excited neutral carbon. Since the intense nickel emission lines lie within the same wavelength regions in which one would expect to detect neutral carbon emission, it is suggested that the failure to resolve the neutral carbon may indicate that this species has a similar velocity and distribution profile to the nickel component within the plume.

\subsection{Plume imaging}

A representative selection of time-gated CCD images of the C-Ni plume at $p(\mathrm{Ar})=5,40,100$ and $340 \mathrm{mT}$ is shown in Figures 6(a) to (d) respectively. Light was collected over a $100 \mathrm{~ns}$ gate width and intensity data accumulated over 25 laser shots with the neutral density filter in place. Each image in Fig. 6 has been contrast-stretched between its maximum (white) and minimum (black) internal values and has been cropped to $800 \times 800$ pixels or $\sim 4.25 \mathrm{~cm}^{2}$. The sequence of images, particularly at 40 and $100 \mathrm{mT}$ as in Figs. 6(b) and (c), reveal three distinct plume emission regions: a broad and fast component at the front of the plume labelled $R 1$; a slower, semi-circular band of emission, $R 2$; and a slow component that remains close to the target over the time period examined, $R 3$. Increasing the background gas pressure clearly confines the plume and at $340 \mathrm{mT}$, Fig. 6(d), there is virtually no spatial separation 
between the leading component $R 1$ and the middle component $R 2$. Neither of these regions is observed at $5 \mathrm{mT}$, Fig. $6(\mathrm{a})$, with only the $R 2 / R 3$ region present in the images.

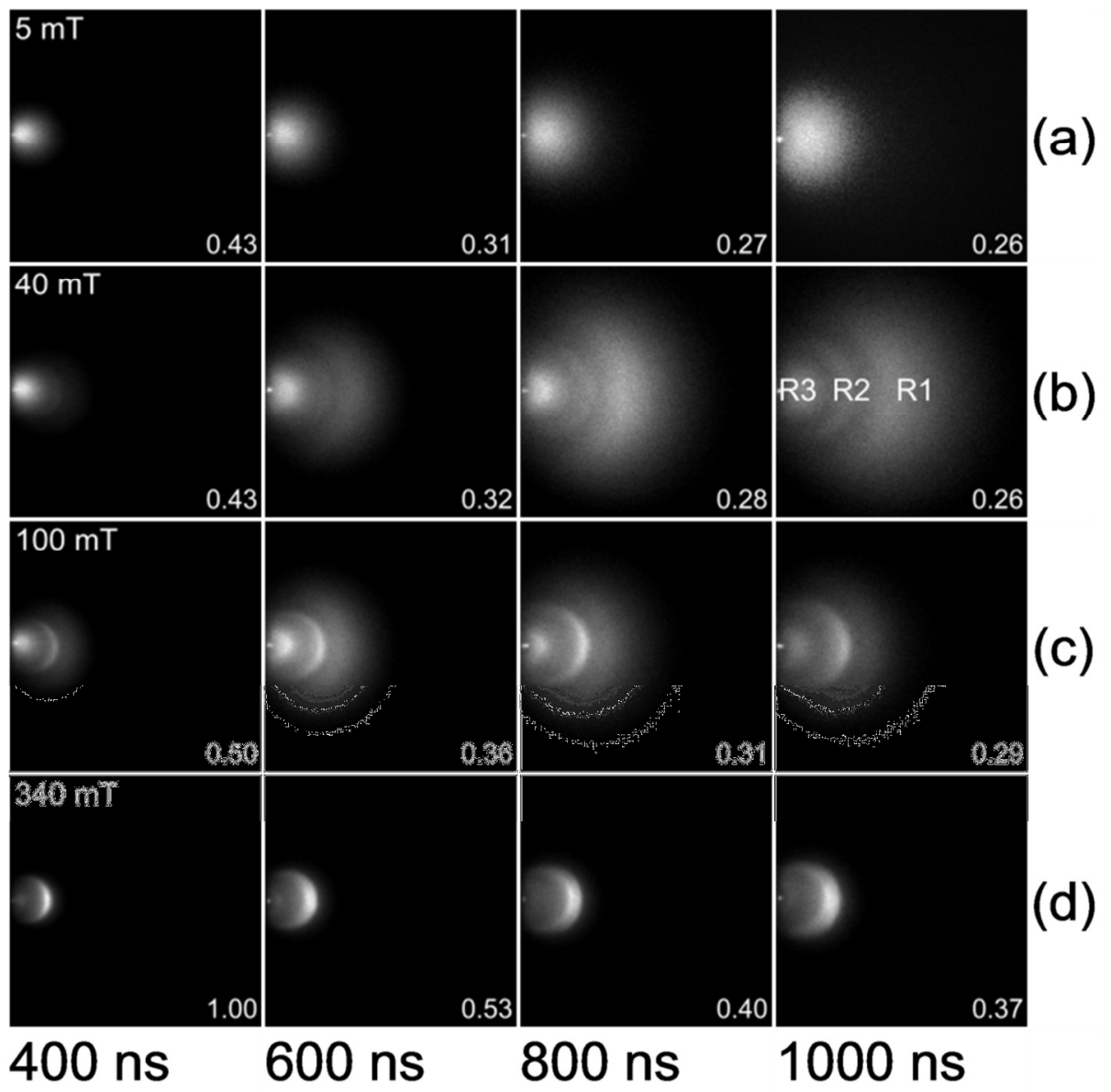

Figure 6: Plume images taken with neutral density filter at (a) 5, (b) 40, (c) 100, and (d) 340 mT background Ar pressures. All images correspond to an area of $4.25 \times 4.25 \mathrm{~cm}$. Fractions in the bottom-right corner of each image represent the relative maximum intensity within the image, represented as white in the greyscale, compared to the image at $400 \mathrm{~ns}$ in $p(\mathrm{Ar})=340$ $\mathrm{mT}(\mathrm{d})$.

The splitting of collisionally-influenced plumes into multiple regions has often been observed in the literature.[37] This "plume-splitting" phenomenon is evident particularly at background pressures in the 40-100 mTorr regime, where the mean-free-path between collisions becomes comparable to the target-substrate distance. Here, the analysis is more 
complex than for the single element systems examined typical in the literature, as the plume contains two different species with different collision cross-sections and masses, producing less clearly-defined and spatially-separated regions. The task here is to determine which species is responsible for the emission in each region and to try and extract useful information regarding their propagation.

To identify the position of nickel emission within the plume, images have been acquired with a $345 \pm 30 \mathrm{~nm}$ band pass filter that transmits the short-wavelength neutral $\mathrm{Ni}$ emission lines observed in the OES of Figs. 4 and 5. Figures 7(a) and (b) show, respectively, neutral density and Ni-filtered images taken $500 \mathrm{~ns}$ after the laser pulse in $p(\mathrm{Ar})=40 \mathrm{mT}$, whereas Figs. 7(c) and (d) are the corresponding images acquired at $1000 \mathrm{~ns}$. This comparison reveals that at early times the slow $R 3$ component correlates with $\mathrm{Ni}$ emission and that the narrow $R 2$ band can also be attributed to nickel emission. The feature marked with an arrow in Fig. 7(c) appears with the neutral density filter but not with the nickel band pass filter - this is likely to be $\mathrm{C}_{2}$ Swan-band emission, as spectroscopically detected in Fig. 5(d) at elevated background gas pressures. 

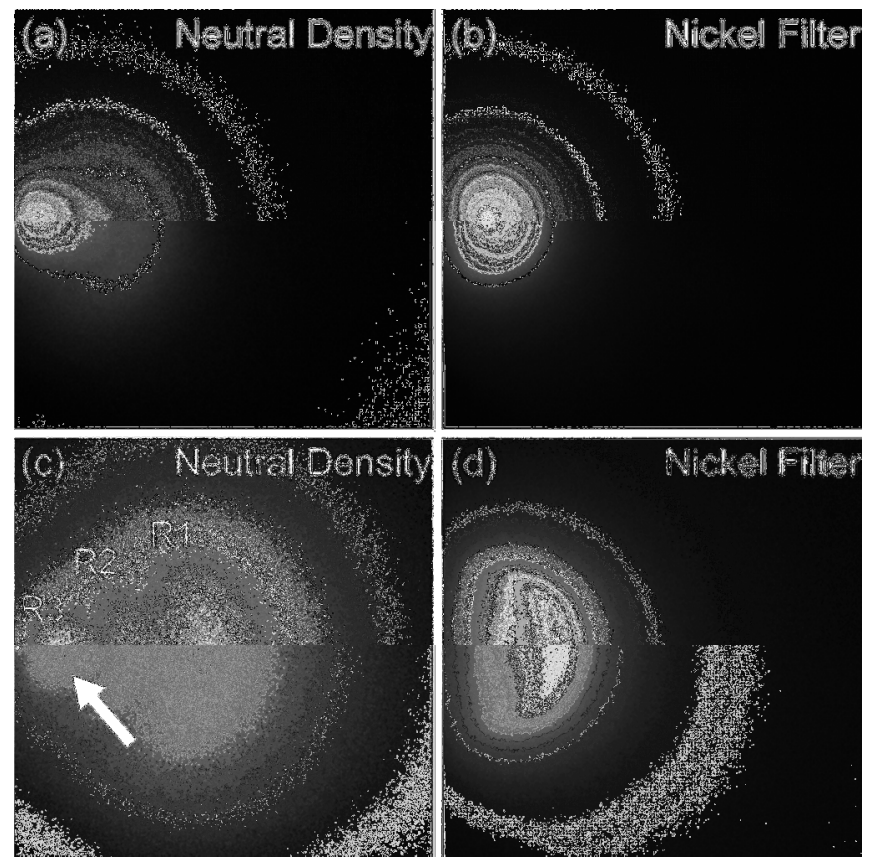

Figure 7: Neutral density and Ni-filtered images of the mixed C-Ni plume with $p(\mathrm{Ar})=40$ $\mathrm{mT}$ at times (a, b) $500 \mathrm{~ns}$ and (c, d) $1000 \mathrm{~ns}$. The nickel-filtered images demonstrate that regions labelled $R 2$ and $R 3$ in Fig. 6 are nickel-related. The feature indicated by an arrow in (c) appears in neutral density, but not nickel-filtered images (d). This is expected to be due to slow moving $\mathrm{C}_{2}$.

The bulk of observable $\mathrm{Ni}$ appears to be relatively slow moving and, early in the plume evolution, gives rise to the $R 3$ emission region close to the target. The $R 2$ region we attribute to collisions between this nickel component and the background argon gas. As the nickel distribution progresses outwards, and its contribution to the intensity near the target diminishes, emission from $\mathrm{C}_{2}$ molecules is detected at later times. One can infer that nickel is not responsible for the broad, fast-moving $R I$ component at the front of the plumes and $R I$ therefore probably involves carbon. This is largely consistent with the estimates of velocities based on OES which suggest a fast carbon component, a trailing nickel component and, at higher pressures, a slow $\mathrm{C}_{2}$ component. 
A number of groups have successfully managed to isolate and image ionised carbon emission on ablation of graphite targets using an appropriate band pass filter $[17,38,39]$. In relation to the mixed target, attempts to do similar were compromised by the failure of such filters to adequately reject the intense nickel lines at elevated background pressures. Here, we are forced to us more complex methods to analyses these mixed plumes. A somewhat contrived solution to this problem was found by filtering the image using a $633 \pm 2 \mathrm{~nm} \mathrm{He}-\mathrm{Ne}$ laser band pass filter, which acts as a 'ramp' filter since it was found to reduce the relative intensity of $\mathrm{Ni}$ emission between 275 and $375 \mathrm{~nm}$, compared to $426.7 \mathrm{~nm}$ (ionised carbon), by roughly an order of magnitude, thus reweighting images in favour of the carbon component.

Figure 8 is a comparison of plume images acquired in vacuum at $700 \mathrm{~ns}$ with (a) the $345 \pm 30$ $\mathrm{nm}$ nickel filter and (b) the $633 \mathrm{~nm}$ 'ramp' filter. The $633 \mathrm{~nm}$ filter reveals a component at the front of the plume that does not appear in the image filtered for nickel emission. Analysis of the images, to be presented below, gives the velocity of its 'centre-of-mass' at $\sim 25 \mathrm{~km} / \mathrm{s}$, in good agreement with the $\sim 30 \mathrm{~km} / \mathrm{s}$ estimate based on the $426.7 \mathrm{~nm}$ emission line in the spatially-resolved OES. It is important to note that the $633 \mathrm{~nm}$ filtered image of Fig. 8(b) is similar to images of a fast-moving ionised/neutral carbon emission region obtained by Geohegan and Puretzky on ablation of graphite [39] with a KrF laser at a fluence of 17.7 $\mathrm{J} / \mathrm{cm}^{2}$. This 'ball of luminescence' was not observed at a fluence of $\sim 6 \mathrm{~J} / \mathrm{cm}^{2}$, equivalent to the fluence used in this study, thereby supporting the observation by OES that the ionised carbon emission is anomalously high given the reduced carbon yield. Since this carbon component is relatively weak compared to nickel emission, one would not expect to observe it at low pressures with the neutral density filter in place and hence images acquired at $5 \mathrm{mT}$, as in Fig. 6(a) previously, show only the $R 2 / R 3$ nickel-related component. The broad and fast emission feature $R l$ observed at higher pressures is therefore attributed to carbon. 

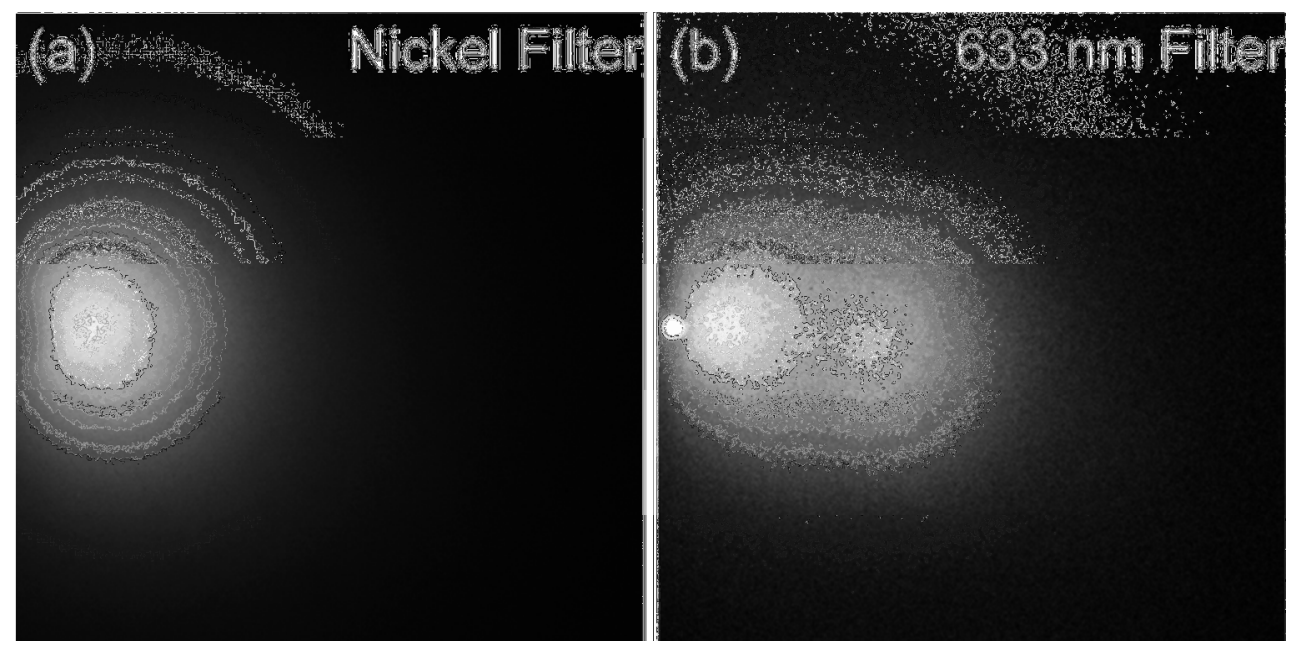

Figure 8: Images of mixed C-Ni plume in vacuum at a time of $700 \mathrm{~ns}$ using (a) the nickel filter and (b) the $633 \mathrm{~nm}$ 'ramp' filter. The use of the $633 \mathrm{~nm}$ filter reduces the relative intensity of nickel and an additional fast-moving plume component is revealed. This feature is attributed to carbon as suggested by plume spectra and velocity data.

\subsection{Velocities and dynamics of plume components}

The dynamics of a plume component in vacuum may be understood in terms of the superposition of two distinct velocity profiles - the adiabatic expansion of the material into vacuum, and a 'flow' velocity away from the target that applies to the centre-of-mass of this expanding component. It is for this reason that velocity data obtained from plumes is often modelled using a shifted Maxwell-Boltzmann distribution [38,40]. In order to extract the flow velocity of the carbon- and nickel-related plume emission regions, intensity profiles have been taken through both neutral density and band pass filtered images. For the nickel component in vacuum, images acquired with the $345 \pm 30 \mathrm{~nm}$ filter were analysed and the profiles fitted to a shifted Maxwell-Boltzmann distribution, as illustrated in Figure 9(a). A similar method using $633 \mathrm{~nm}$ 'ramp' filtered images was used to extract the velocity of the ionised carbon component in vacuum. In each case the position of the relevant component as 
a function of time was taken as the peak, or intensity maximum, of the corresponding fitting curve. The position of the $R 2$ nickel region in the presence of a background gas was determined from profiles of nickel-filtered images, Fig. 9(b), using a pair of Lorentzian curves to distinguish between the bright region at the front and emission from the trailing distribution. The position of the fast, carbon-related $R l$ region was taken from neutral density filtered images and extracted by considering the emission profile to be composed of three overlapping Lorentzian distributions corresponding to carbon, nickel, and nickel/ $\mathrm{C}_{2}$ emission regions, as in Fig. 9(c). As with the vacuum profile data, the intensity maxima of the fitting curves were used to determine the position of the corresponding plume component.

This analysis is expected to yield the velocity of the centre of the emission distribution or, in other words, the flow velocity of the corresponding emitting species in vacuum. However, with the introduction of the background gas, extraction of the position of intensity maxima may not necessarily yield a comparable result. The previous OES and imaging data indicates that the emission intensity of plume species with $p(\mathrm{Ar})=340 \mathrm{mT}$ is approximately twice that observed in vacuum. This enhancement in the presence of the background gas may therefore distort the intensity distribution in the images relative to the vacuum case. Furthermore, since the plume is confined with increasing gas pressure there is also the possibility that emission from one species will begin to overlap with that of another thereby raising the relative intensity in these regions. One must therefore address the question as to what the maximum intensity in the image profiles represents when plumes have been examined in the presence of the background gas.

Figure 10(a) shows an image of the $\mathrm{C}-\mathrm{Ni}$ plume taken with the neutral density filter at $\mathrm{t}=$ $1000 \mathrm{~ns}$ and $p(\mathrm{Ar})=40 \mathrm{mT}$. The extent of visible carbon-related $R 1$ and nickel-related $R 2$ 
emission in the image is illustrated by the appropriately-labelled circular boundary. In the case of the carbon-related $R I$ region, the emitting species appear to have a broad velocity distribution but the intensity maxima is roughly located in the centre of that distribution. We conclude that by taking the intensity maxima of the $R l$ region, the analysis subsequently yields the flow velocity of this carbon emission region. This does not appear to be the case for the Ni component. The extent of the circular boundary surrounding the $R 2$ region in Figure 10(a) is also consistent with images filtered for Ni. From this we infer that the analysis will yield the position, and hence velocity, of the $\mathrm{Ni} / \mathrm{Ar}$ boundary or the forward edge of the Ni region. Based on the circular symmetry, one might reasonably estimate that the flow velocity of the emitting $\mathrm{Ni}$ species will be approximately one-half that determined from our analysis. Figure 10(b) is the same image as Fig. 10(a) but with the intensity minimum clipped at $40 \%$ of maximum to highlight the regions of high intensity utilised in the extraction of velocities. 


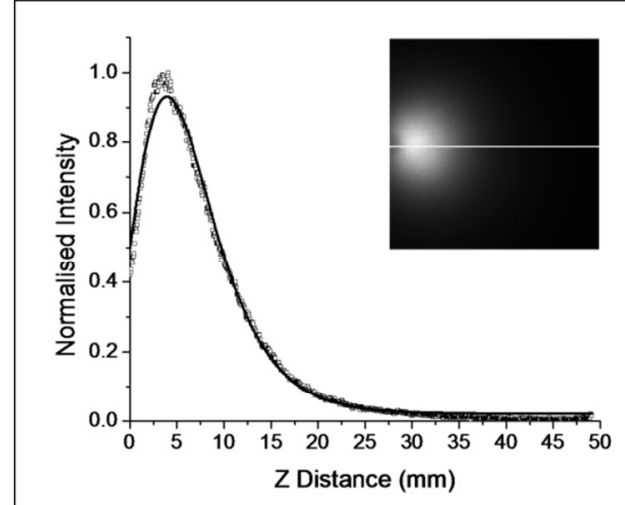

(a)

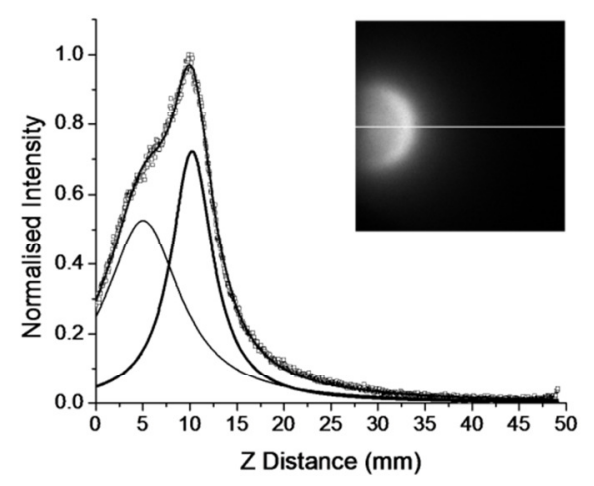

(b)

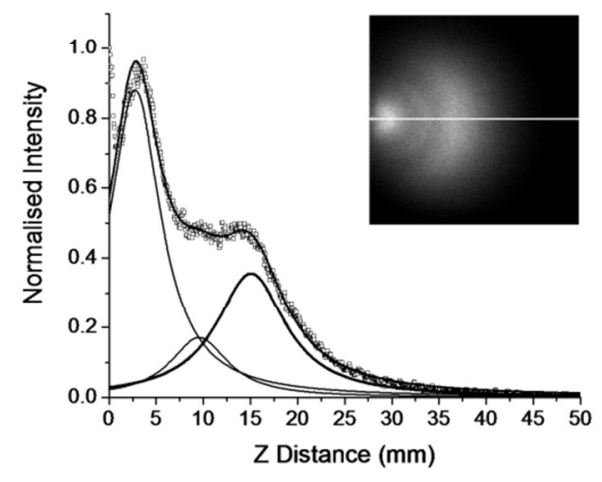

(c)

Figure 9: Representative profiles through images in the propagation direction of the plume. In (a) profiles from nickel-filtered images in vacuum have been fit using a shifted MaxwellBoltzmann distribution; (b) profiles from nickel-filtered images of the plume in elevated pressures have been fit to two Lorentzian functions to isolate the position of the $\mathrm{Ni} / \mathrm{Ar}$ interface from the trailing distribution of nickel, and (c) the position of the carbon-related region determined by fitting neutral density filtered image profiles with three Lorentzian functions. 

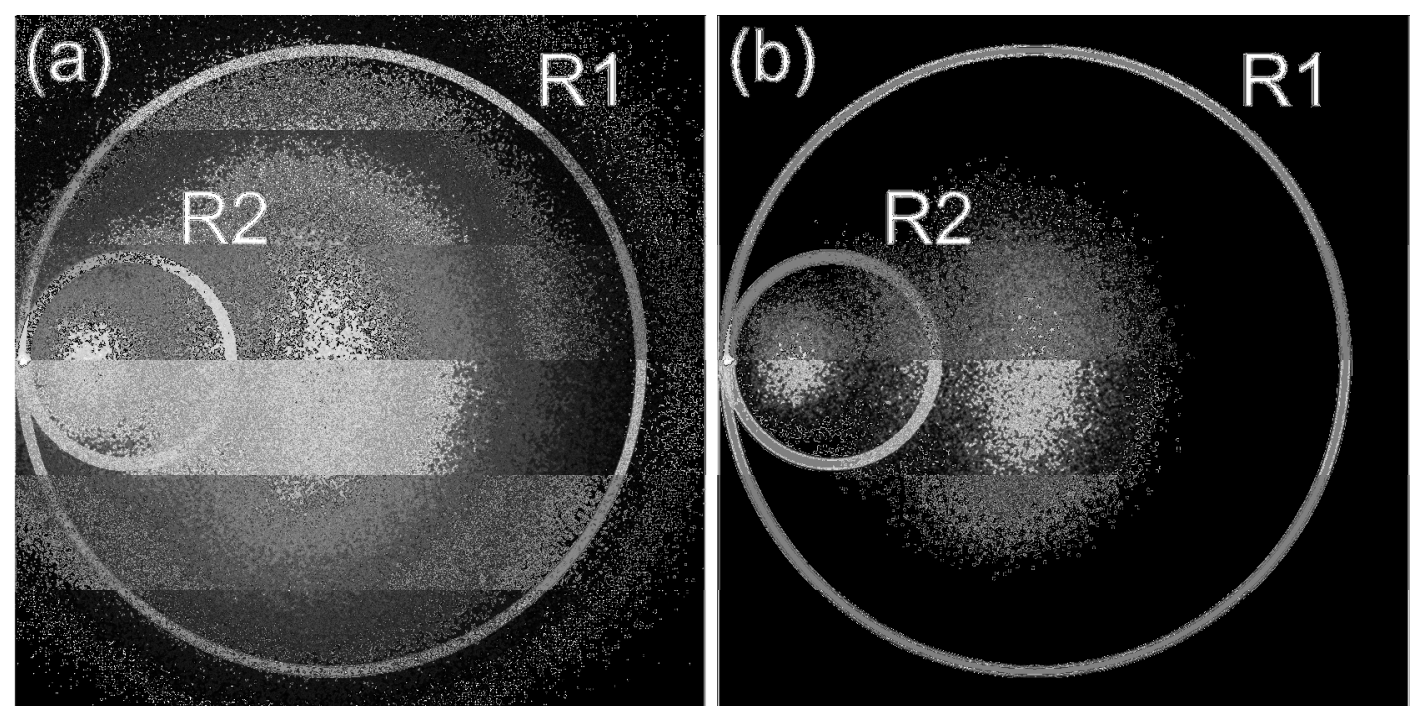

Figure 10: Illustration of the spatial extent of carbon- and nickel-related emission in the mixed plume at $\mathrm{t}=1000 \mathrm{~ns}$ with $p(\mathrm{Ar})=40 \mathrm{mT}$. Each image represents an area of $\sim 4.25 \mathrm{~cm}^{2}$. In (a) the image has been contrast stretched between the minimum and maximum plume intensity values, whereas (b) has been clipped at $40 \%$ of maximum. The intensity maxima determined from intensity profiles along the propagation direction are likely to yield the flow velocity of the $R 1$ C-related component (corresponding to the centre of the circular distribution), and the velocity of the Ni/Ar interface in the case of $R 2$ (corresponding to the forward edge of the nickel distribution).

The resulting position data for vacuum, $p(\mathrm{Ar})=40,100$ and $340 \mathrm{mT}$ are shown in Figures 11(a) to (d), respectively. The flow velocity of the fast carbon component in vacuum, Fig. 11(a), is determined to be $\sim 24.7 \mathrm{~km} / \mathrm{s}$ while the nickel velocity is $\sim 6.8 \mathrm{~km} / \mathrm{s}$. In both cases the values are close to those estimated from the plume emission spectra. At $40 \mathrm{mT}$, Fig. 11(b), the $R 1$ carbon region moves with an initial flow velocity close to that in vacuum $(\sim 24 \mathrm{~km} / \mathrm{s})$ and rapidly decelerates at times greater than $1000 \mathrm{~ns}$. The initial velocity of the $R 2$ nickel component at $40 \mathrm{mT}$ is $\sim 10 \mathrm{~km} / \mathrm{s}$. Although this appears higher than the velocity in vacuum $(6.8 \mathrm{~km} / \mathrm{s})$, the emission at $R 2$ represents the $\mathrm{Ni} / \mathrm{Ar}$ boundary and hence is due to emission of material at the leading edge of the nickel distribution. The subsequent deceleration of this $R 2$ 
region is less marked than for the carbon region. The behaviour of each component at 100 mT, Fig. 11(c), is broadly comparable to that at $40 \mathrm{mT}$ with somewhat contracted stopping distances for both regions and, when the pressure is increased to $340 \mathrm{mT}$, Fig. 11(d), the carbon-related $R 1$ and Ni-related $R 2$ regions move in tandem with a spatial separation of a only a few millimetres between them.

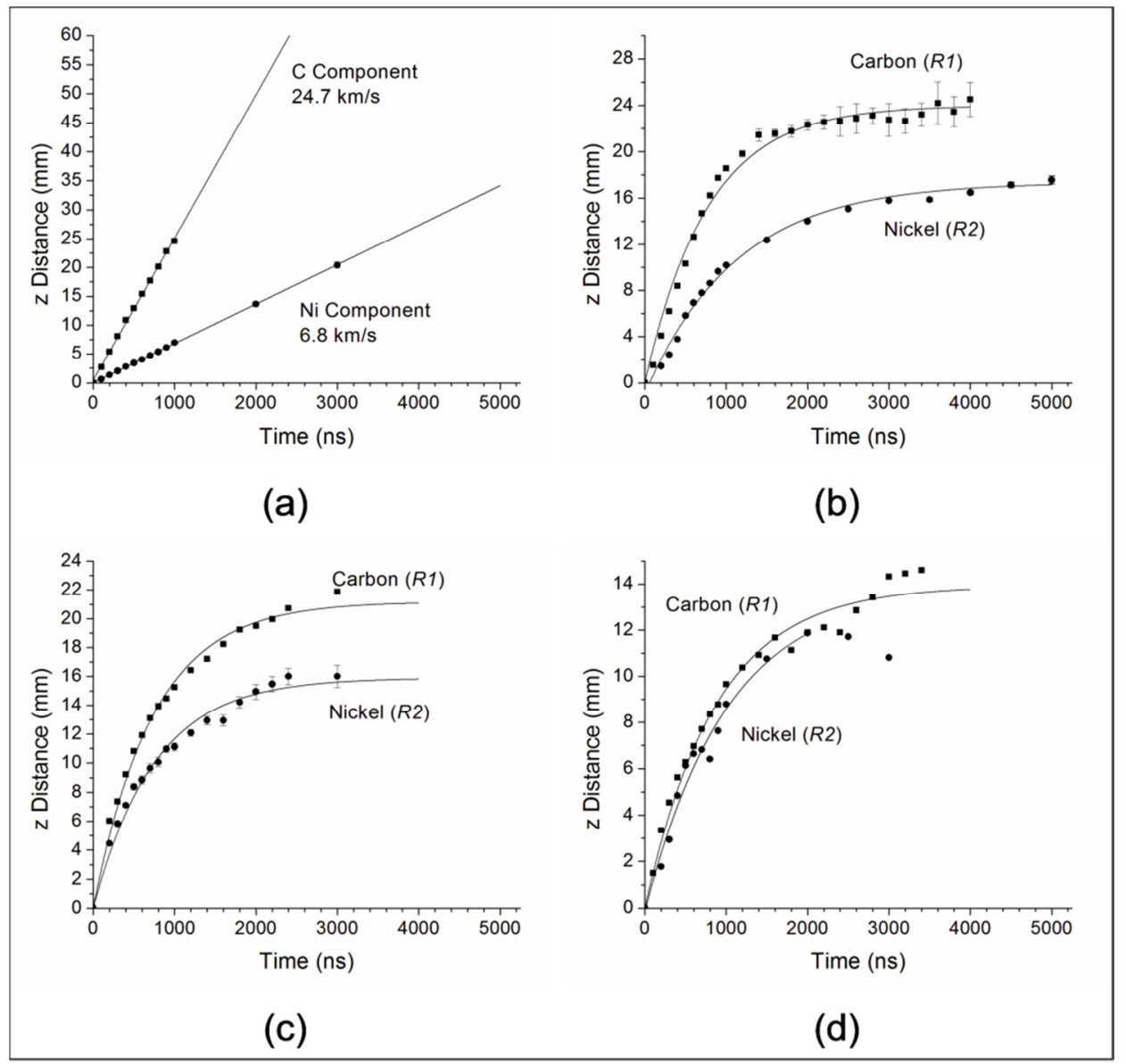

Figure 11: Distance versus time plots based on image profile data for plume components in (a) vacuum, (b) $40 \mathrm{mT}$, (c) $100 \mathrm{mT}$, and (d) $340 \mathrm{mT}$ background pressures. Curves in (b) to (d) are fits to the 'drag model' of plume propagation, with fitting parameters shown in Table 1.

The data of Fig. 11 at $p(\mathrm{Ar})=40 \mathrm{mT}$, particularly at early times in the plume evolution, is not accurately modelled by applying the so-called 'shockwave' equation [41] where the 
propagation distance $z \propto t^{n}$. This indicates that at low pressures the density of background gas is insufficient for shockwave formation. The plume images observed in this region show the evidence of plume-splitting, where the material that has collided appears spatially partitioned. Although this model does fit the position data at 100 and $340 \mathrm{mT}$ reasonably well, with values of $n$ between 0.4 and 0.5 , it does not provide the necessary comparison of the plume propagation characteristics at each pressure. Instead, the fitting curves shown in Figs. 11(b) to (d) were generated using the drag model $z=z_{f}[1-\exp (-\beta t)]$, where $z_{f}$ is the 'stopping distance', $t$ is time, and $\beta$ is the 'slowing coefficient' [42]. Parameters to achieve the best fits are shown in Table 1 . The slowing coefficients determined at 40, 100 and 340 $\mathrm{mT}$ for the fast $\mathrm{C}$ region are interchangeable and qualitatively good fits are achieved regardless of whether one uses the $1.31,1.25$ or $1.14 \times 10^{6} \mathrm{~s}^{-1}$ values. This is also the case for the $\mathrm{Ni}$ slowing coefficients at each pressure. One can nevertheless conclude that the coefficients for the $\mathrm{C}$ region are higher than for the Ni region since qualitatively good fitting is not generally achieved by interchanging the values for the two components.

Table 1: Fitting parameters of drag model $z=z_{f}[1-\exp (-\beta t)]$, where $z_{f}$ is the stopping distance, $t$ is time, and $\beta$ is the slowing coefficient, at 40,100 , and $340 \mathrm{mT}$.

\begin{tabular}{|c|c|c|c|}
\hline $\begin{array}{c}\text { Pressure } \\
(\mathrm{mT})\end{array}$ & Component & $\begin{array}{c}\mathrm{z}_{\mathrm{f}} \\
(\mathrm{mm})\end{array}$ & $\begin{array}{c}\beta \\
\left(\times 10^{6} \mathrm{~s}^{-1}\right)\end{array}$ \\
\hline 40 & $\mathrm{C}(R l)$ & $23.9(3)$ & $1.31(6)$ \\
& $\mathrm{Ni}(R 2)$ & $18.3(14)$ & $0.89(15)$ \\
\hline 100 & $\mathrm{C}(R l)$ & $20.6(6)$ & $1.25(8)$ \\
& $\mathrm{Ni}(R 2)$ & $15.4(4)$ & $1.01(8)$ \\
\hline 340 & $\mathrm{C}(R 1)$ & $13.9(3)$ & $1.14(7)$ \\
& $\mathrm{Ni}(R 2)$ & $13.9(11)$ & $0.99(12)$ \\
\hline
\end{tabular}


The data of Fig. 11 and Table 1 demonstrate that the deceleration of the carbon component, as a function of pressure, is more pronounced than for nickel. An analogous result is obtained when one examines the expansion rate of the plume. Figure 12 shows the radius of the carbon and nickel components at (a) $40 \mathrm{mT}$ and (b) $340 \mathrm{mT}$ perpendicular to the propagation direction. The dimensions in each case were extracted using a similar method to the velocities above, with profiles taken across filtered and unfiltered images and fit to a Lorentzian function. The profile position was chosen to coincide with the widest part of the associated emission region which, in the case of the $\mathrm{Ni}$ component, does not lie through the region of maximum intensity utilised in Fig. 11. The value plotted is the width encompassing $85 \%$ of the total emission intensity of the profile, and may be conceptualised as being broadly equivalent to the radius of the circular boundary as illustrated in Fig. 10.

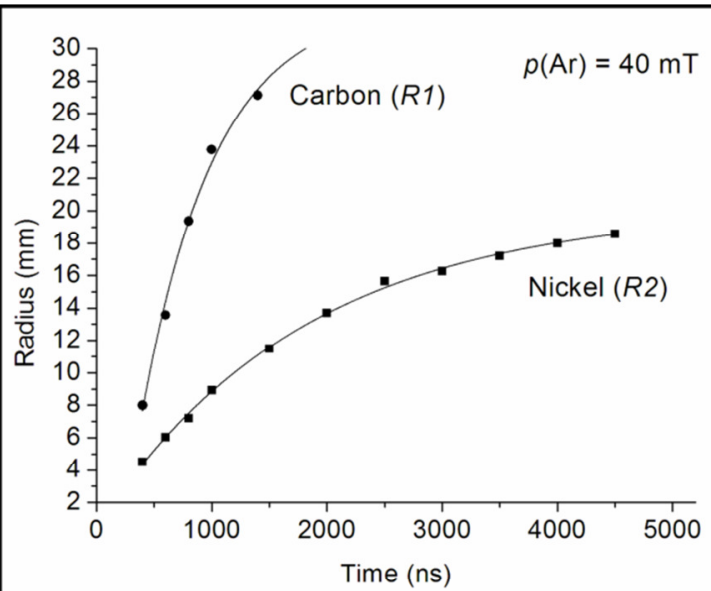

(a)

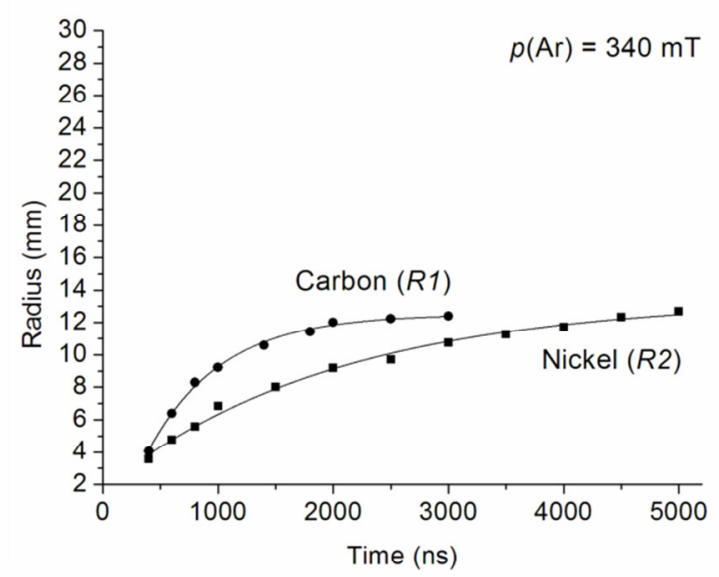

(b)

Figure 12: Comparison of the radius of $\mathrm{C}$ - and $\mathrm{Ni}$ - related emission regions perpendicular to the propagation direction at $\mathrm{p}(\mathrm{Ar})=40$ (a) and 340 (b) $\mathrm{mT}$. Curves in each case are fits to the 'drag model' of plume propagation, with fitting parameters shown in Table 2. 
As with the velocities of Fig. 11, the radius as a function of time of each component has been fit using the drag model and parameters are shown in Table 2. At $40 \mathrm{mT}$, the radius of the Crelated $R l$ emission region has a stopping distance of $\sim 47 \mathrm{~mm}$, which is approximately twice the $\sim 24 \mathrm{~mm}$ stopping distance for the centre-of-mass of the distribution (Table 1.) The stopping distance for the radius of the Ni-related $R 2$ region $(\sim 19.7 \mathrm{~mm})$ is roughly comparable to that determined from the position of the maximum of intensity along the propagation direction in Table $1(18.3 \mathrm{~mm})$, confirming the prior assessment that the $R 2$ region represents the edge of the Ni distribution. At $340 \mathrm{mT}$, the stopping distance of both the C- and Ni-radii is comparable to the values in Table 1, suggesting a substantial braking of the C-component. As above, it is found that the slowing coefficients for $\mathrm{C}$ are significantly higher than for $\mathrm{Ni}$.

Table 2: Fitting parameters of drag model $z=z_{f}[1-\exp (-\beta t)]$ to plume radius, where $z_{f}$ is the stopping distance, $t$ is time, and $\beta$ is the slowing coefficient, at 40 and $340 \mathrm{mT}$.

\begin{tabular}{|c|c|c|c|}
\hline $\begin{array}{c}\text { Pressure } \\
(\mathrm{mT})\end{array}$ & Component & $\mathrm{z}_{\mathrm{f}}$ & $\beta$ \\
\hline 40 & $\mathrm{C}(R l)$ & $46.9(37)$ & $1.57(41)$ \\
& $\mathrm{Ni}(R 2)$ & $19.7(3)$ & $0.56(3)$ \\
\hline 340 & $\mathrm{C}(R l)$ & $15.8(6)$ & $1.58(1)$ \\
& $\mathrm{Ni}(R 2)$ & $11.7(3)$ & $0.50(5)$ \\
\hline
\end{tabular}

We apply the following simple model, as illustrated in Figure 13, in order to explain the changing $\mathrm{C}: \mathrm{Ni}$ ratio found in thin films as a function of the background gas pressure. It is 
assumed, for the moment, that the $\mathrm{C}$ and $\mathrm{Ni}$ in the plume are confined within the $R 1$ and $R 2$ regions respectively. In the case of carbon species, the stopping distances of Table 1 represent the final position after deceleration of the centre of the distribution. Similarly, based on the arguments above, it is assumed that the centre of the Ni distribution is located at half the distance quoted in Table 1.

Two points were considered at a distance equal to the centre of the $\mathrm{Ni}$ and $\mathrm{C}$ distributions at a given pressure, and the initial nickel-to-carbon ratio chosen to reflect the $52: 48$ at $\%(\sim 1.08)$ observed in samples grown at $5 \mathrm{mT}$ or less. It was then assumed that each component can be represented by a sphere centred at these points with a radius equal to the distance to the substrate, shown as $L_{1}$ and $L_{2}$ in Fig. 13. The fraction of nickel per unit area of sphere is then simply determined to be $52 \times 4 \pi L_{1}^{2}$ and, similarly, $48 \times 4 \pi L_{2}^{2}$ for carbon. The ratio of these values was taken as an estimate of the $\mathrm{Ni}: \mathrm{C}$ ratio arriving at the substrate and the results of this calculation for 40, 100 and $340 \mathrm{mT}$ are shown in Figure 14, along with the ratios determined by EDX at $x, y=0$ for reference. Although obviously contrived, this simple model qualitatively replicates the decrease and subsequent increase of the observed $\mathrm{Ni}$ fraction in films as a function of $p(\mathrm{Ar})$, and suggests this is due to the difference in the confinement effect of the background gas on carbon and nickel species.[7,43] The main discrepancy occurs at 40 mTorr, which is likely due the assumption that of all the species are stopping as a single distribution (i.e. with little plume splitting) is less valid. This model does not account for the effect of clustering in the plume, but it would be expected that more Ni rich clusters form in regions where the $\mathrm{Ni}$ atom density is higher (back of the plume) and carbon rich clusters form at the front. The diffusion (transport) of these mixed clusters then occurs with the material at the front having less distance to diffuse. 


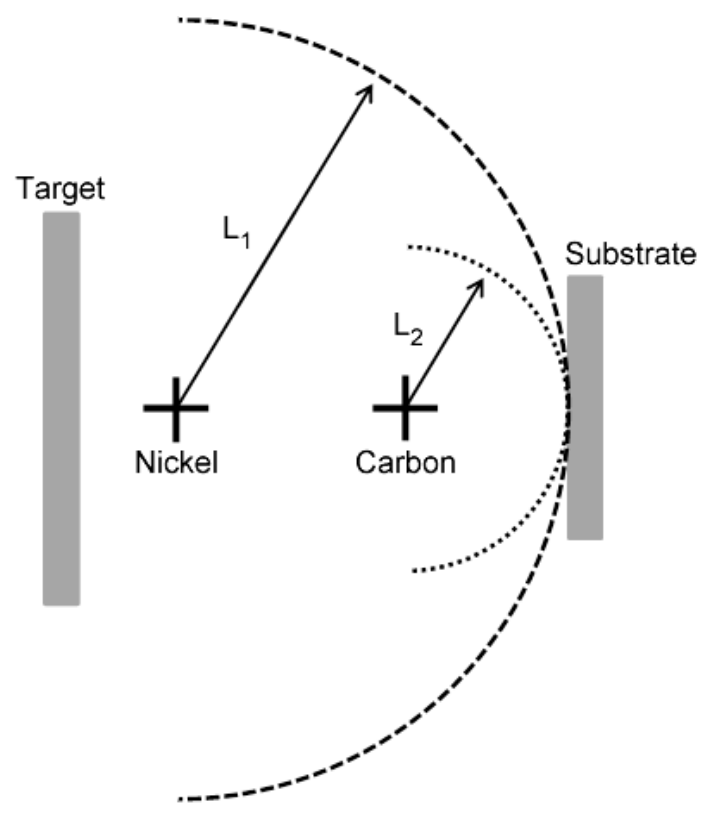

Figure 13: Illustration of the effect of stopping distances on the relative $\mathrm{Ni}: \mathrm{C}$ ratio in substrates. It is assumed that the stopping distances of Table 1 represent the centre of the $\mathrm{C}$ distribution. The centre of the Ni distribution is taken as half the value indicated in Table 1 . The relative concentration of material arriving at the substrate is then related to the lengths $\mathrm{L}_{1}$ and $\mathrm{L}_{2}$.

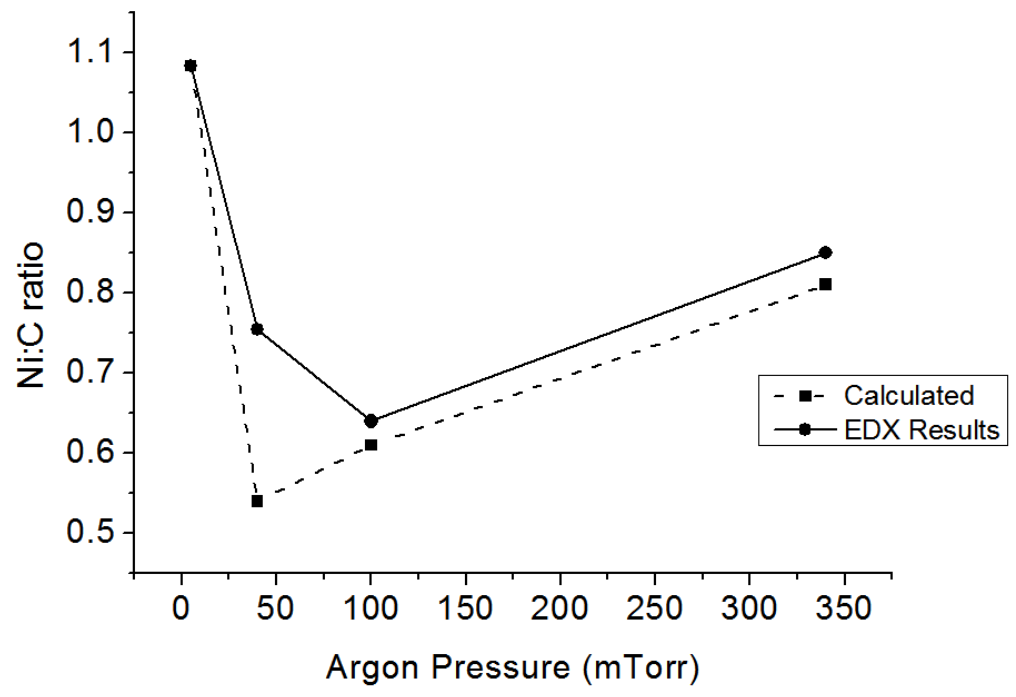

Figure 14: Graph showing calculated and measured Ni:C ratio in samples as a function of pressure based on the model of Fig. 13. 
We therefore propose that the behaviour of the plume as a function of $p(\mathrm{Ar})$ can be divided into three distinct phases.

(1) At pressures $\leq 5 \mathrm{mT}$, the background gas has little effect on the plume dynamics. Carbon and nickel species removed from the target arrive at the substrate and the resulting $\mathrm{C}: \mathrm{Ni}$ fraction in the films is representative of the target yield, which becomes increasingly nickelrich with successive laser shots until some stable value is reached.

(2) As the background gas pressure is increased to $40 \mathrm{mT}$ and above, carbon and nickel species become increasingly confined. Plume splitting partitions the material into spatially separated regions. Combining the data of Tables 1 and 2, the stopping distance of the fast edge of the $R l$ carbon-related component at $p(\mathrm{Ar})=40 \mathrm{mT}$ is larger than the target-tosubstrate distance $(23.9+46.9>60 \mathrm{~mm})$. This is not the case for the $R 2$ Ni-related component $(\sim 19 \mathrm{~mm})$ at $40 \mathrm{mT}$. Since Ni clearly arrives at the substrate, one can consider that the transfer of material becomes increasingly 'diffusion-like' with increasing gas pressure and species must propagate with some (at least partially) thermalized velocity [7, 40].

The $\mathrm{C}: \mathrm{Ni}$ ratio in films is then determined by the relative spatial positions of the centre of $\mathrm{C}$ and Ni distributions as suggested by the simple model of Figs. 13 and 14. Although plume imaging is only sensitive to emitting species, we infer that this mechanism must also apply to weaker or non-emitting material in the plume - provided a significant fraction of the carbon, whether visible or not, has a flow velocity approximately equivalent to that of $\mathrm{Ni}$, an increase in the carbon fraction in the films will result as the carbon component is increasingly confined. This argument is consistent with our failure to observe a separation of neutral carbon emission and nickel emission in both the OES and imaging data. 
(3) At pressures above $100 \mathrm{mT}$, the majority of material in the plume is thermalized close to the target and diffusion becomes the dominant mechanism by which material arrives at the substrate. Since the diffusion distance to the substrate is roughly equivalent for carbon and nickel species, the C:Ni ratio in films approaches that found under vacuum growth.

\section{Conclusions}

Pulsed-laser deposition using a $\mathrm{KrF}$ laser is found to produce thin films in vacuum and low pressure conditions in which the $\mathrm{C}$ : Ni ratio deviates significantly from the relative proportion of those elements in a mixed carbon-nickel target. We have proposed that a nickelenrichment of the target surface occurs as a result of both a preferential back-scattering of nickel near the ablation zone and, to some extent, to a slower cooling of the porous material of the mixed target leading to outwards metal diffusion. The high nickel fraction found in films is attributed to the re-ablation of this surface metal coating with successive laser shots leading possibly to a stable surface stoichiometry.

The nickel content of films is found to decrease as a function of background Ar pressure up to $100 \mathrm{mT}$ and recover at pressures of $340 \mathrm{mT}$. Optical emission spectroscopy and imaging reveal an ablation plume that contains slow nickel- and fast carbon-related regions, and analysis of the velocity and distribution of these components as a function of background pressure suggests that the stopping distances are generally closer to the target for nickel than for carbon, except at $340 \mathrm{mT}$ when both components overlap. A simple model has been proposed whereby the $\mathrm{C}: \mathrm{Ni}$ ratio is ultimately dependent on the difference in distance required for thermalized carbon-rich and nickel-rich material (clusters) to reach the substrate. These results have significant implications for the understanding of the synthesis of carbon nanomaterials, such as carbon nanotubes, and for carbon/metal alloy films by pulsed laser ablation, as the metal content in the ablated plume may evolve over significant time-scales 
during the synthesis. The mechanisms discussed can be generalised for a wide range of mixed targets, but the carbon/metal case can be considered as a particularly extreme version as the masses, atom sizes and vapour pressures of the two elements differ significantly.

\section{Acknowledgements}

The authors would like to thank Prof. Mike Ashfold, Dr. Gareth Fuge and Ben Truscott of the University of Bristol for many valuable discussions and suggestions. This work was funded under EPSRC grant number EP/FO52901/1.

\section{References}

[1] Jayawardena KDGI, Tan YY, Fryar J, Shiozawa H, Silva SRP, Henley SJ, et al. Highly conductive nanoclustered carbon:nickel films grown by pulsed laser deposition. Carbon. 2011;49: 3781-3788.

[2] Rode AV, Gamaly EG, Luther-Davies B. Formation of cluster-assembled carbon nanofoam by high-repetition-rate laser ablation. Appl. Phys. A - Mater. 2000;70:135-144.

[3] Milani P, Podestà A, Piseri P, Barborini E, Lenardi C, Castelnovo C. Cluster assembling of nanostructured carbon films. Diam. Relat. Mater. 2001;10:240-247.

[4] Rode AV, Hyde ST, Gamaly EG, Elliman RG, McKenzie DR, Bulcock S. Structural analysis of a carbon foam formed by high pulse-rate laser ablation. Appl. Phys. A - Mater. 1999;69:S755-S758.

[5] Bongiorno G, Podestà A, Ravagnan L, Piseri P, Milani P, Lenardi C, et al. Electronic properties and applications of cluster-assembled carbon films. J. Mater. Sci. - Mater. El. 2006; 17:427-441. 
[6] Rode AV, Elliman RG, Gamaly EG, Veinger AI, Christy AG, Hyde ST, et al. Electronic and magnetic properties of carbon nanofoam produced by high-repetition-rate laser ablation. Appl. Surf. Sci. 2002;197: 644-649.

[7] Schou J. Physical aspects of the pulsed laser deposition technique: The stoichiometric transfer of material from target to film. Appl. Surf. Sci. 2009;255: 5191-5198.

[8] Orgiani P, Ciancio R, Galdi A, Amoruso S, Maritato L. Physical properties of $\mathrm{La}_{0.7} \mathrm{Ba}_{0.3} \mathrm{MnO}_{3-\delta}$ complex oxide thin films grown by pulsed laser deposition technique. Appl. Phys. Lett. 2010;96: 023501-3

[9] Arnold CB, Aziz MJ. Stoichiometry issues in pulsed-laser deposition of alloys grown from multicomponent targets. Appl. Phys. A - Mater. 1999;69: S23-S27.

[10] Acquaviva S, De Giorgi ML. Stoichiometric analyses of $\mathrm{Cd}_{1-\mathrm{x}} \mathrm{Mn}_{\mathrm{x}} \mathrm{Te}$ film deposition by pulsed laser ablation. Appl. Surf. Sci. 2003;208-209: 620-625.

[11] Dam B, Rector JH, Johansson J, De Groot DG, Griessen R. Non-Stoichiometric Transfer of Complex Oxides by Pulsed Laser Deposition at Low Fluences. MRS Proceedings, 1995;397: 175 doi:10.1557/PROC-397-175.

[12] Gonzalo J, Afonso CN, Perrière J, Gómez San Roman R. The importance of gas scattering processes on the stoichiometry deviations of laser deposited films. Appl. Surf. Sci. 1996;96-98: 693-696.

[13] van de Riet E, Kools JCS, Dieleman J. Incongruent transfer in laser deposition of FeSiGaRu thin films. J. Appl. Phys. 1993;73: 8290-8296.

[14] Anisimov SI, Luk'yanchuk BS, Luches A. An analytical model for three-dimensional laser plume expansion into vacuum in hydrodynamic regime. Appl. Surf. Sci. 1996;96-98: 24-32.

[15] Toftmann B, Schou J, Lunney JG. Dynamics of the plume produced by nanosecond ultraviolet laser ablation of metals. Phys. Rev. B. 2003;67:104101-104105. 
[16] Droubay TC, Qiao L, Kaspar TC, Engelhard MH, Shutthanandan V, Chambers SA. Nonstoichiometric material transfer in the pulsed laser deposition of $\mathrm{LaAlO}_{3}$. Appl. Phys. Lett. 2010;97:124105.

[17] Henley SJ, Carey JD, Silva SRP, Fuge GM, Ashfold MNR, Anglos D. Dynamics of confined plumes during short and ultrashort pulsed laser ablation of graphite. Phys. Rev. B $2005 ; 72: 205413$.

[18] Henley SJ, Carey JD, Silva SRP. Room temperature photoluminescence from nanostructured amorphous carbon. Appl. Phys. Lett. 2004;85:6236-6238.

[19] Lifshitz Y, Kasi SR, Rabalais JW. Subplantation model for film growth from hyperthermal species: application to diamond. Phys. Rev. Lett. 1989;62:1290-1293.

[20] Norton DP. Pulsed Laser Deposition of Complex Materials: Progress Towards Applications. In: Pulsed Laser Deposition of Thin Films: Applications-Led Growth of Functional Materials. Eason R, editor. New Jersey:Wiley; 2007, p. 5.

[21] Puretzky AA, Geohegan DB, Jellison Jr. GE, McGibbon MM. Comparative diagnostics of ArF- and KrF-laser generated carbon plumes used for amorphous diamond-like carbon film deposition. Appl. Surf. Sci. 1996;96-98: 859-865.

[22] Pestchanyi SE, Landman IS. Effective thermal conductivity of graphite materials with cracks. Phys. Scripta 2004;T111:218-229.

[23] Lutkov AI. Effect of porosity of the effective thermal conductivity of graphite. J. Eng. Phys. Thermophys. 1973;25(2):1042-1044. Translated from Inzhenerno-Fizicheskii Zhurnal $1973 ; 25(2): 325-329$

[24] Kudryashov SI, Tikhov AA, Zvorykin VD. Near-critical nanosecond laser-induced phase explosion on graphite surface. Appl. Phys. A - Mater. 2010;102: 493-499.

[25] Xu X, Song K. Interface kinetics during pulsed laser ablation. Appl. Phys A - Mater. 1999;69: S869-S873. 
[26] Claeyssens F, Cheesman A, Henley SJ, Ashfold MNR. Studies of the plume accompanying pulsed ultraviolet laser ablation of zinc oxide. J. Appl. Phys. 2002;92: 68866894.

[27] Claeyssens F, Henley SJ, Ashfold MNR. Comparison of the ablation plumes arising from ArF laser ablation of graphite, silicon, copper, and aluminium in vacuum. J. Appl. Phys. 2003;94:2203-2211.

[28] Shinozaki T, Ooie T, Yano T, Yoneda M. Diagnostics of KrF- and Nd:YAG-laser produced carbon plumes by time- and spatially-resolved spectroscopy. Jpn. J. Appl. Phys. 2000;39:6272-6276.

[29] Kushwaha A, Thareja RK. Dynamics of laser-ablated carbon plasma: formation of $\mathrm{C}_{2}$ and CN. Appl. Optics 2008;47:G65-G71.

[30] Nemes L, Keszler AM, Parigger CG, Hornkohl JO, Michelsen HA, Stakhursky V. Spontaneous emission from the $C_{3}$ radical in carbon plasma. Appl. Optics 2007;46:40324040.

[31] Arepalli S, Nikolaev P, Holmes W, Scott CD. Diagnostics of laser-produced plume under carbon nanotube growth conditions. Appl. Phys. A - Mater. 1999;69:1-9.

[32] Arepalli S, Scott CD. Spectral measurements in production of single-wall carbon nanotubes by laser ablation. Chem. Phys. Lett. 1999;302:139-145.

[33] Song KH, Xu X. Explosive phase transformation in excimer laser ablation. Appl. Surf. Sci. 1998;127-129:111-116.

[34] Pappas DL, Saenger KL, Cuomo JJ, Dreyfus RW. Characterization of laser vaporization plasmas generated for the deposition of diamond-like carbon. J. Appl. Phys. 1992;72:39663970.

[35] Trimmer C, Srivastava SK, Hall TE, Fucaioro AF. Enhanced line emission from laserproduced plasmas. J. Appl. Phys. 1991;70:1888-1890. 
[36] Abdelli-Messaci S, Kerdja T, Bendib A, Aberkane SM, Lafane S, Malek S. Investigation of carbon plasma species emission at relatively high $\mathrm{KrF}$ laser fluences in nitrogen ambient. Appl. Surf. Sci. 2005;252: 2012-2020.

[37] Wood RF, Leboeuf JN, Geohegan DB, Puretzky AA, Chen KR, Dynamics of Plume Propagation and Splitting During Pulsed Laser Ablation of Si in He and Ar, Phys. Rev. B 1998;58:1533-1543.

[38] Claeyssens F, Lade RJ, Rosser KN, Ashfold MNR. Investigations of the plume accompanying pulsed ultraviolet laser ablation of graphite in vacuum. J. Appl. Phys. 2001;89:697-709.

[39] Geohegan DB, Puretzky AA. Species-resolved imaging and gated photon counting spectroscopy of laser ablation plume dynamics during KrF- and ArF-laser PLD of amorphous diamond films. Mater. Res. Soc. Symp. P. 1996;397:55-68.

[40] Kools JCS, Baller TS, De Zwart ST, Dieleman J. Gas flow dynamics in laser ablation deposition. J. Appl. Phys. 1992;71: 4547-4556.

[41] Zel'dovich YB, Raizer YP. Physics of Shock Waves and High-Temperature Hydrodynamic Phenomena. New York:Academic; 1996.

[42] George S, Kumar A, Singh RK, Nampoori VPN. Effect of ambient gas on the expansion dynamics of plasma plume formed by laser blow off of thin film. Appl. Phys. A - Mater. 2010;98:901-908.

[43] Itina TE, Marine W, Autric M. Monte Carlo simulation of pulsed laser ablation from two-component target into diluted ambient gas. J. Appl. Phys. 1997;82:3536-3542. 